UCRL-CR-120519

\title{
The Determination of the Constitutive Parameters of a Medium with Application to a Reinforced Concrete Pad
}
A. J. Poggio
G. J. Burke

S. T. Pennock

January 15, 1995

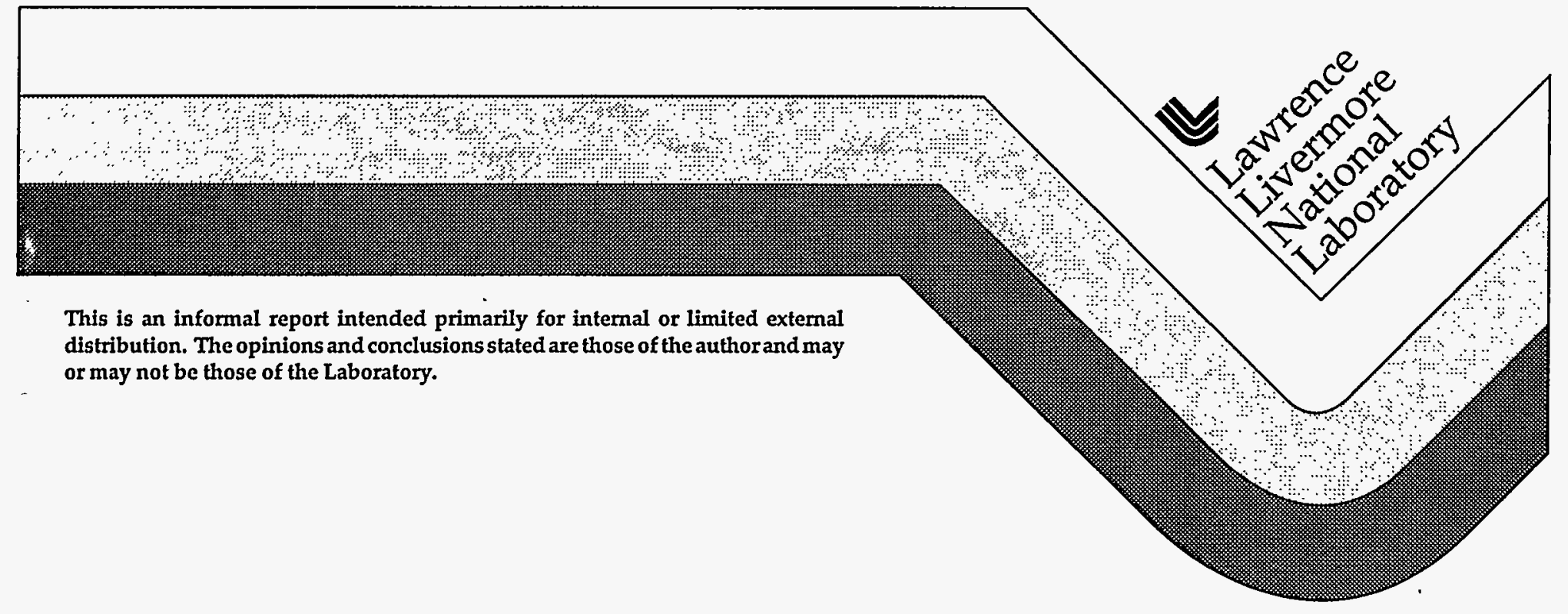


This document was prepared as an account of work sponsored by an agency of the United States Government. Neither the United States Government nor the University of California nor any of their employees, makes any warranty, express or implied, or assumes any legal liability or responsibility for the accuracy, completeness, or usefulness of any information, apparatus, product, or process disclosed, or represents that its use would not infringe privately owned rights. Reference herein to any specific commercial products, process, or service by trade name, trademark, manufacturer, or otherwise, does not necessarily constitute or imply its endorsement, recommendation, or favoring by the United States Government or the University of California. The views and opinions of authors expressed herein do not necessarily state or reflect those of the United States Government or the University of California, and shall not be used for advertising or product endorsement purposes.

This report has been reproduced directly from the best available copy.

Available to DOE and DOE contractors from the Office of Seientific and Technical Information

P.O. Box 62, Oak Ridge, TN 37831

Prices avaitable from (615) 576-8401, FTS 626-6401.

Avaitable to the public from the National Technical Information Service

U.S. Department of Commerce 5285 Port Royal Rd.,

Springfield, VA 22161

Work performed under the auspices of the U.S. Department of Energy by the Lawrence Livermore National Laboratory under Contract W-7405-Eng-48. 


\section{DISCLAIMER}

Portions of this document may be illegible in electronic image products. Images are produced from the best available original document. 


\title{
The Determination of the Constitutive Parameters of a Medium with Application to a Reinforced Concrete Pad
}

\author{
A. J. Poggio \\ G. J. Burke \\ S. T. Pennock \\ Lawrence Livermore National Laboratory
}

January 15,1995 


\section{CONTENTS}

Acknowledgment

Introduction

Proposed Approach

Solutions for a Wire Over Ground

Physical Layout

Measurement Process

Experiment and Data

Acquired Data

Extraction of Attenuation and Phase Constants

3

4

6

6

8

8

Determination of Relative Dielectric Constant and Conductivity

Analysis

Conclusions and Summary

General Comments

Data Summary

Concerns and Suggested Future Work 


\section{Acknowledgment}

The authors are grateful to a number of individuals for their support during the performance of the experimental program. The Test Director was Ly Dao of USAF Phillips Laboratory and the experimental operations team, led by John Millard of United International Engineering Inc., included James Tucker of UIE.

This effort was part of the NASA Low Power On-the-Ground Test portion of the NASA Boeing 757 HIRF Tests under Project Manager Charles Meissner. This, in turn, was part of the NASA Fly-By-Light/Power-by-Wire Program. 


\section{Introduction}

This report describes the experimental and analytical program performed to determine the constitutive parameters of the reinforced concrete pad in the test facility used during the Low Power On-the-Ground portion of the NASA Boeing 757 HIRF Tests [1]. These tests were conducted during the period September 20 to October 21, 1994 in the LESLI facility at the Phillips Laboratory, Kirtland AFB, NM.

The on-the-ground tests were designed to meet several objectives including support of a flight test series and the generation of data for the validation of codes and models that could be used to predict the electromagnetic environment in transport aircraft. To satisfy these objectives, tests were to be executed in a known environment and the data compared to modeling results. A critical feature of this testing was the "known environment" which implies knowledge of the parameters which are critical to an effective modeling activity and which could include, among many other things, definitions of the airplane and its physical and electrical configuration, the ground upon which it sits when stationary, the fields impinging on the aircraft, and the radiating or bounding structure in the simulator. We would want to specify the electromagnetic characteristics of the entire space that would likely enter into a mathematical modeling effort so that the model can be made as "close" to physical reality as desired prior to exercising computational algorithms which might introduce their own uncertainties.

Since we are evaluating codes used for determination of the electromagnetic environment in aircraft and since the on-the-ground test involved an airplane parked on a pad in the simulator facility, we would require a definition of the constitutive parameters of the pad. Initially, the test plan called for the pad to be partially covered by a highly conducting covering composed of interconnected aircraft landing mats with a total surface area in excess of $15,000 \mathrm{ft}^{2}$. Difficulties associated with timely delivery of the mats, installation of the panels on the pad, and uncertainties in the quality of the electrical interconnection of the individual panels prevented use of these panels and required situation of the airplane on the bare, reinforced concrete pad. As a result, the need for measurement of the constitutive parameters of the LELSI concrete pad arose. 
The evaluation of the constitutive parameters was performed under a number of significant constraints that served to restrict the available options. The constraints were:

- In order to limit costs, the measurement process was to be minimized in time extent without severely compromising the quality of the data

- The measurement process was limited by the availability of the experimental system including the RF sources and the data acquisition system. Ultimately, this limitation confined the pad characterization tests to a one-week period including assembly and disassembly of the test fixtures and equipment, testing and dry runs of the entire system, equipment down-time, and actual acquisition runs for the data.

- Construction details for the pad, constructed over 40 years ago, were not available. Thus, reinforcing rod location, concrete thickness and specifications were unknown.

- The measurement techniques were to be completely noninvasive, namely, the pad could not be cored or probes emplaced below the surface.

- The RF equipment and the data acquisition team's experience in performing ground parameter measurements were somewhat limited. Accurate reflection angle, scattering and polarization measurements as might be required in Brewster angle and wave tilt measurements were possibly beyond the capabilities of the available equipment.

Since the precise detailed structure of the pad is unknown and since a detailed spatial description of the constitutive parameters is not required for the purposes of the code validation exercise in view of the level of precision with which other environmental descriptors are known, a technique which averages material characteristics over some spatial extent has been deemed adequate. In view of these observations, the constraints above, and discussions with experts in this technical area such as Drs. Ray King and George Hagen, a method based on wave propagation along wires closely coupled to the ground structure was chosen. The remainder of this report describes the test and analyses involved in determining the constitutive parameters. In the analysis, arguments are made regarding the adequacy of the quality of the results insofar as their role in the code validation exercise is concerned. It would not.be reasonable to expend inordinate resources to greatly reduce uncertainties in the parameters when the uncertainties might only be reflected as second order effects. But, realizing the potential value of this scheme for the determination of constitutive parameters in general situations, some concerns and suggestions for future woík are presented. 


\section{Proposed Approach}

The method chosen for the determination of the constitutive parameters is based on the theoretical solution for current propagation on a wire above a homogeneous half-space with arbitrary permittivity $\varepsilon$, and conductivity $\sigma$. For the purposes of this study, the permeability $\mu$ of both half spaces (above and below the ground plane) will be assumed equal to that of free space $\mu_{0}$. A solution for the current distribution on an infinitely long wire over ground, with no limitations on the ground constants, has been developed by Chang, Olsen and Kuester [2, 3, 4]. Their analysis leads to a modal expansion of the current on the infinitely long wire in which they found two discrete modes and continuous spectrum components associated with radiation. One of the discrete modes is referred to as the structure attached or transmission line mode, is dominant near the source on a wire close to the ground, and is a slow wave in the case of conducting grounds. The other discrete mode exhibits fast wave characteristics, has substantially less attenuation along the direction of the wire than does the transmission line mode, and is mainly important for large heights above ground.

\subsection{Solutions for a Wire Over Ground}

In [2], with time variation exp[-i $\omega$ t], an integral equation for the current on an infinite horizontal wire along the $\mathrm{x}$ axis, with radius a and height $\mathrm{z}=\mathrm{h}$ over a ground plane, is solved for a delta-function source located at $\mathrm{x}=0$. The solution for the current is

$$
I(x)=\frac{2}{\pi \zeta_{o}} \int_{-\infty}^{\infty} \frac{\exp (i k x \gamma)}{\mathrm{M}(\gamma)} d \gamma
$$

where $M(\gamma)$ is the Fourier transform of the integral equation kernel and $k=\left(\mu_{0} \varepsilon_{0}\right)^{1 / 2}$, is the propagation constant in the upper half-space. For a perfectly conducting wire, $M(\gamma)$ is [4]

$$
\mathrm{M}(\gamma)=\zeta^{2}\left[H_{o}^{(1)}(\zeta A)-J_{o}(\zeta A) H_{o}^{(1)}(2 \zeta H)\right]+P(\gamma, 2 H)-\gamma^{2} Q(\gamma, 2 H)
$$

where $\mathrm{H}=\mathrm{kh}$ and $\mathrm{A}=\mathrm{ka}$, and $\zeta=\left(1-\gamma^{2}\right)^{1 / 2}$ with $\operatorname{Im} \zeta \geq 0$. The functions $\mathrm{P}$ and $\mathrm{Q}$ are given by the integrals

$$
P(\gamma, Z)=\frac{2}{i \pi} \int_{-\infty}^{\infty} \frac{e^{-u_{1} Z}}{u_{1}+u_{2}} d \lambda \quad Q(\gamma, Z)=\frac{2}{i \pi} \int_{-\infty}^{\infty} \frac{e^{-u_{1} Z}}{n^{2} u_{1}+u_{2}} d \lambda
$$


where $n=\left(\varepsilon_{r}+i \sigma / \omega \varepsilon_{0}\right)^{1 / 2}$ for $\varepsilon_{r}$ and $\sigma$ the relative dielectric constant and conductivity of the ground and

$$
\begin{array}{ll}
u_{1}=\left(\lambda^{2}-\zeta^{2}\right)^{1 / 2}, & u_{2}=\left(\lambda^{2}-\zeta_{n}^{2}\right)^{1 / 2}, \quad \operatorname{Re} u_{1}, u_{2} \geq 0 \\
\zeta_{n}=\left(n^{2}-\gamma^{2}\right)^{1 / 2}, & \operatorname{Im} \zeta_{n} \geq 0 .
\end{array}
$$

The current in Equation (1) is represented as a spectrum of exponential waves with a propagation constant $\mathrm{k} \gamma$. However, if the integration contour is deformed into the complex . $\gamma$ plane, the current is found to be composed of one or two discrete modes at poles of the integrand where $M(\gamma)=0$ and three branch cut integrals [4]. For the wire height and frequencies considered here, the important components are a discrete mode with $\gamma=\gamma_{p}$, where $M\left(\gamma_{p}\right)=0$, and a branch cut integral from $\gamma=1$. This discrete mode has the character of an attenuating slow wave and is called the transmission line mode. The component of current in this mode is given by the residue at the pole as

$$
\mathrm{I}_{\mathrm{p}}(\mathrm{x})=4 \mathrm{i}\left(\mu_{0} \varepsilon_{0}\right)^{-1 / 2} \exp \left(\mathrm{ik} \gamma_{\mathrm{p}} \mathrm{x}\right) / \mathrm{M}^{\prime}\left(\gamma_{\mathrm{p}}\right)
$$

There can be a second discrete mode, known as the surface-attached mode, which has the nature of a fast wave, but it does not appear significant in the case of our measurements. The branch cut integral yields a component of current with asymptotic form $\exp (\mathrm{ikx}) / \mathrm{x}^{2}$, so it is important very near the source but it quickly drops below the transmission line mode. However, at some larger distance the exponential decay of the transmission line mode will always take it below the branch cut term which is decreasing algebraically. Hence, there is a window in $\mathrm{x}$ where the transmission line mode is dominant, and this region is of primary interest in our measurements since information about the ground parameters is most easily extracted from $\gamma_{p}$.

In the typical forward solution for the discrete modes, the ground constants $\varepsilon$ and $\sigma$ are substituted into Equation (2) and the solution for $M(\gamma)=0$ is found by numerical iteration. For the problem at hand, namely determination of $\varepsilon_{\mathrm{r}}$ and $\sigma$ given the attenuation and phase constant for a measured current distribution on the wire, the inverse problem must be solved. Formally, for a given frequency, wire radius, and height above ground, $\alpha$ and $\beta$ are determined by fitting the measured current $I(x)$. Then $\gamma_{p}=(\beta+i \alpha) / k$ is substituted for $\gamma$ in Equation (2) and the equation is solved numerically for $\varepsilon_{\mathrm{r}}$ and $\sigma$. The final results are then the constitutive parameters for the pad at a set of frequencies (as many as desired). 


\subsection{The Physical Layout}

The concrete pad that is the subject of this analysis is shown in Figure 1. The pad, which is approximately 94 meters in diameter, is composed of reinforced concrete with unknown structural characteristics. The source region is covered with an aluminum plate as shown, which measures approximately 20 meters from the edge of the pad to the triangular plates forming the transition region from aluminum to concrete. For the purposes of the characterization, one of the LESLI rhombic antenna wires was stretched, under some tension, across the pad and continued across the earth surroundings. The wire, with . diameter $3 / 16$ inches (radius $=0.238 \mathrm{~cm}$ ), was approximately 200 meters in length with the portion beyond the 100 meter mark containing randomly placed loads in an attempt to suppress reflections when used in the rhombic configuration. In an attempt to isolate the wire from the pad over a considerable portion of its length, the wire was placed on a dielectric support. The closed cell Styrofoam support was composed of two strips each $1 / 4$ inch thick and 8 inches wide to form a $1 / 2$ inch by 8 inch support structure. The support was secured to the concrete with duct tape. A cross section view of the arrangement of the wire, Styrofoam support, aluminum plane, and concrete pad is shown in Figure 2. The Styrofoam support material was characterized using a network analyzer and a stripline fixture, and its relative dielectric constant was measured to be approximately 1.03 over the range of frequencies from $50 \mathrm{MHz}$ to $1 \mathrm{GHz}$. The wire was tensioned with turnbuckles and secured to a stake beyond the edge of the concrete pad. The remainder of the wire, containing the randomly located loads, was allowed to remain on the ground in the random configuration it assumed when lowered to the ground. Cutouts were made in the Styrofoam support strip to accommodate the current probes. Ultimately, the axis of the wire was $5 / 8$ inches $(1.5875 \mathrm{~cm}$ ) with an uncertainty of $1 / 8$ inch above the ground plane. The current was measured with Prodyne 1320 current probes that were connected to NanoFast high frequency fiber optic links by one-meter long coaxial cables that were orthogonal to the wire to minimize coupling and high frequency losses. The signals were routed to a fiber optic ( $\mathrm{F}-\mathrm{O}$ ) receiver for optical to electrical conversion and then to an HP automatic network analyzer. Ultimately the network analyzer output was routed to a recording computer. The system is shown in Figure 3.

\subsection{The Measurement Process}

To execute the tests, the wire was driven against the aluminum ground plane and the current distribution was measured at selected points along the wire. From this measured 


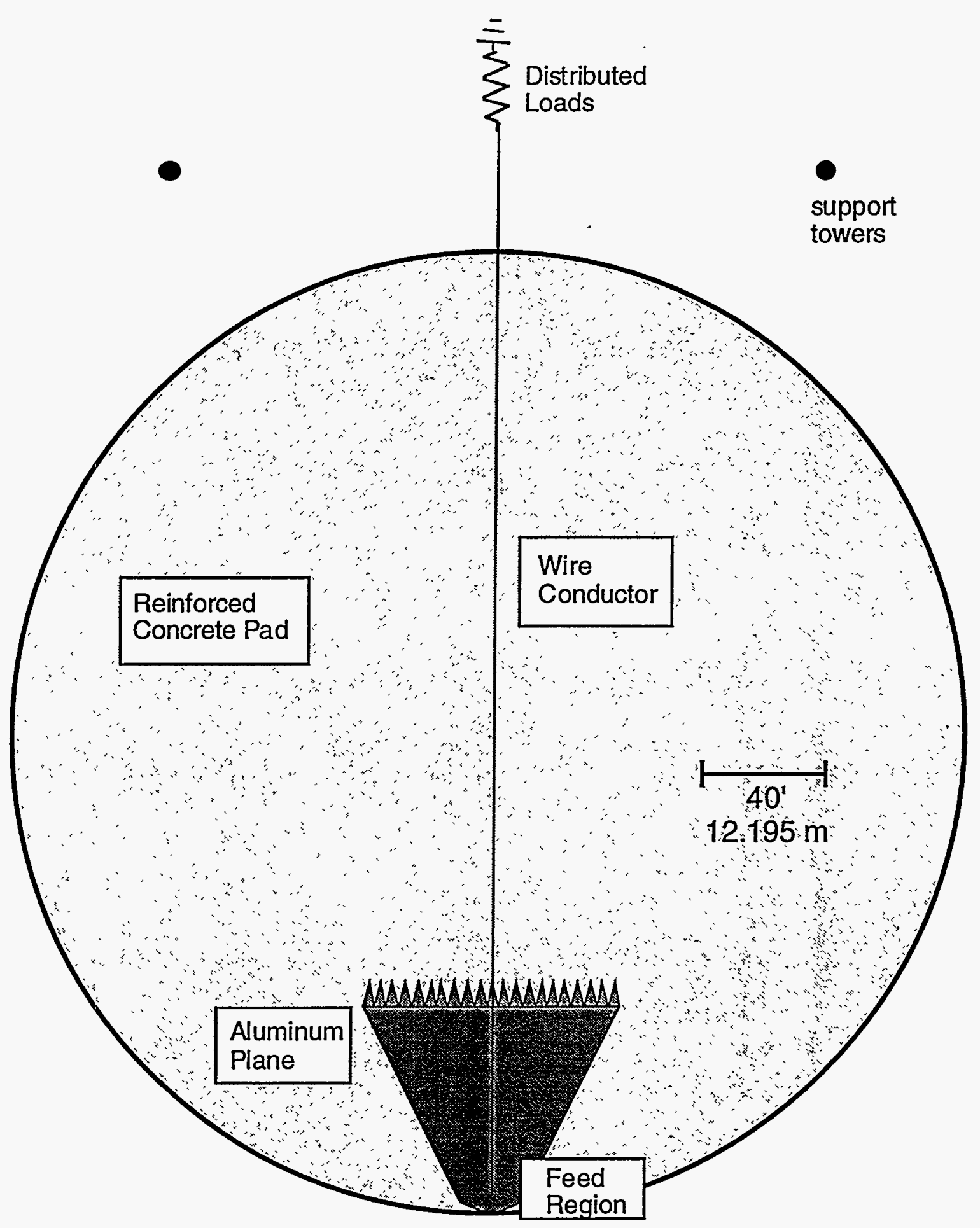

Figure 1. Pad characterization wire layout 


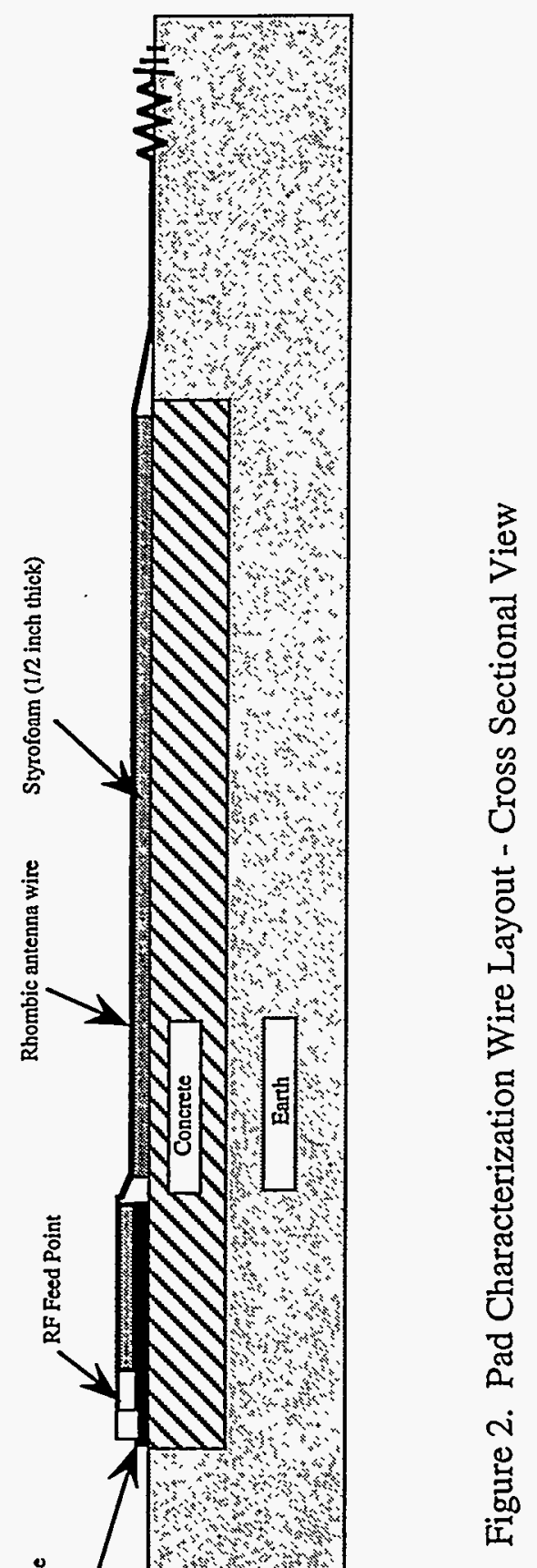




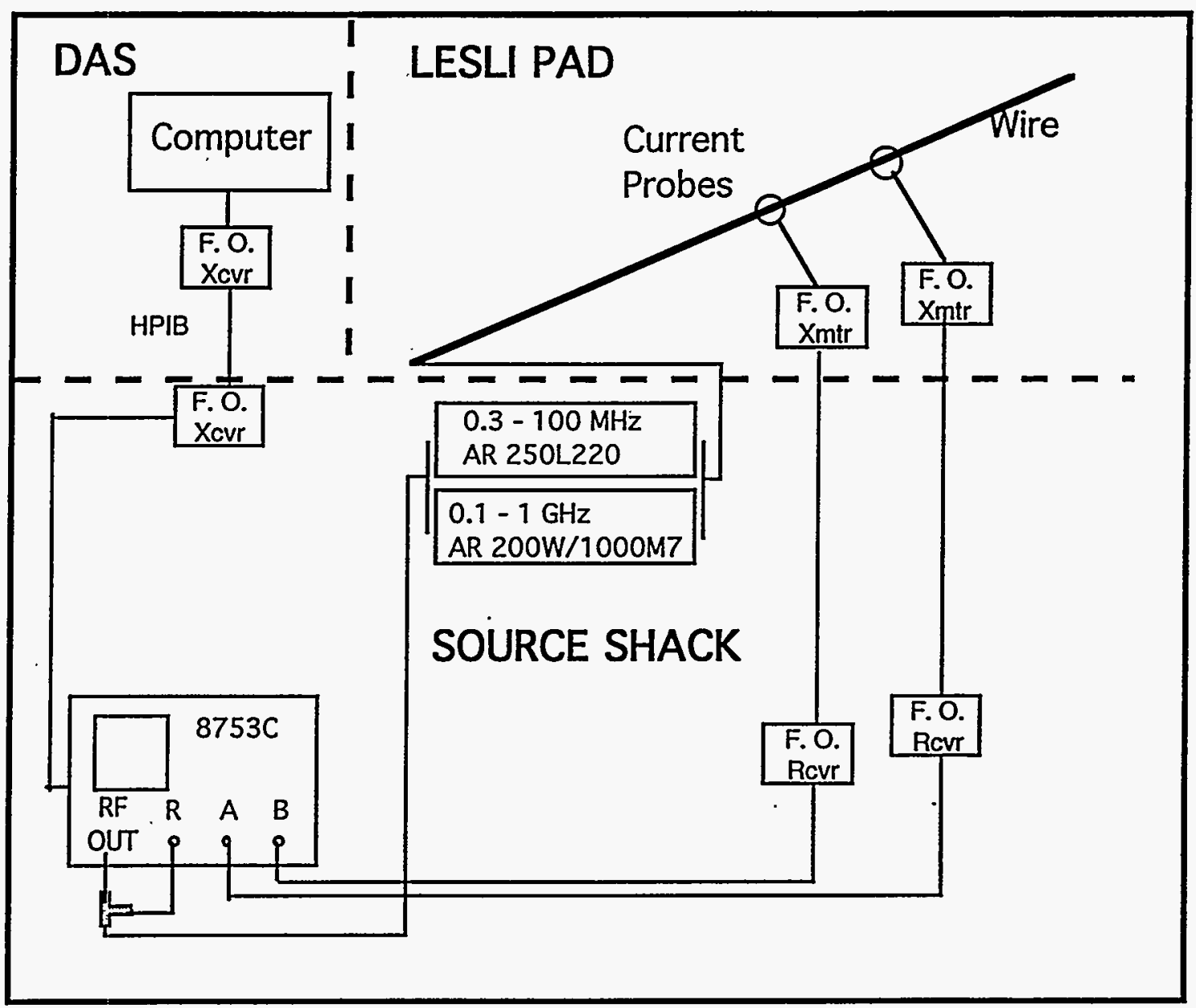

Figure 3. Ground characterization instrumentation 
current distribution the attenuation and phase constants were determined using methods described later. The method used in data acquisition was to simultaneously measure the current at two points along the structure over the entire range of frequencies with one point (Point 1) always located at the first measurement site closest to the source, viz., $x=22.5$ meters. For the purposes of this study, the frequency range was from approximately 1 $\mathrm{MHz}$ to approximately $1 \mathrm{GHz}$. Starting at a point 22.5 meters from the feed point on the aluminum plane that coincides with the rhombic apex, the current was measured at $30 \mathrm{~cm}$ intervals for a total of 90 points. The locations, with the reference probe at 22.5 meters (Point 1 ), are given by

$$
\mathrm{x}(\text { meters })=22.5+0.3(\mathrm{n}-1), \quad \mathrm{n}=\{1,90\}
$$

Based on the premise that the current distribution for a wire very close to the ground is dominated, especially near the source, by a complex exponential exp(ik $\gamma \mathrm{x}$ ) with $\gamma$ defined by zeroes of $\mathrm{M}(\gamma)$ in the complex plane, it becomes clear that relative measurements of current are adequate for evaluation of attenuation and phase constants. For example, the ratio of current at Point $\mathrm{n}$ to the current at Point 1 when the currents are measured simultaneously, or are the results of identical source conditions at the feedpoint, is simply

$$
\mathrm{I}\left(\mathrm{x}_{\mathrm{n}}\right) / \mathrm{I}\left(\mathrm{x}_{1}\right)=\exp \left(\mathrm{ik} \gamma\left(\mathrm{x}_{\mathrm{n}}-\mathrm{x}_{1}\right)\right)
$$

Clearly the reference signal strength is normalized out of the analysis and evaluation of the attenuation and phase constant becomes straightforward, in principle. The data was collected by stepping through all frequencies of interest in a spatial-pairwise scheme $\Pi\left(x_{n}\right)$ and $\left.I\left(x_{1}\right)\right]$ for all $n$. Hence, we measure and store $I\left(x_{n}, \omega_{j}\right) / I\left(x_{1}, \omega_{j}\right), n=\{2,90\}$, and $j=\{1, J\}$, i.e., we record the normalized current (as well as each component, in reality) at fixed positions for stepped frequency sweeps. Ultimately, we use this data in a different format, namely, we use the normalized current at fixed frequency for stepped position to determine $\alpha$ and $\beta$. The structure of this data matrix is represented graphically in Figure 4 where $I\left(x_{i}, \omega_{j}\right)$ is represented by $I_{i j}$ and the data is acquired along columns (fixed position) and processed along rows (fixed frequency). This then allows the determination of the normalized attenuation and phase constants as a function of frequency. Using these, one then performs the inverse evaluation of the constitutive parameters as described later. Some uncertainties arise regarding this approach to the determination of the constitutive parameters. Namely, it is unknown, a priori, if this method is robust and unaffected by the presence of noise in the measured data. The literature abounds with non-robust methods for evaluation of the parameters in complex exponentials. In addition to the impact of measurement noise in the data, the impact of other uncertainties such as the importance of 


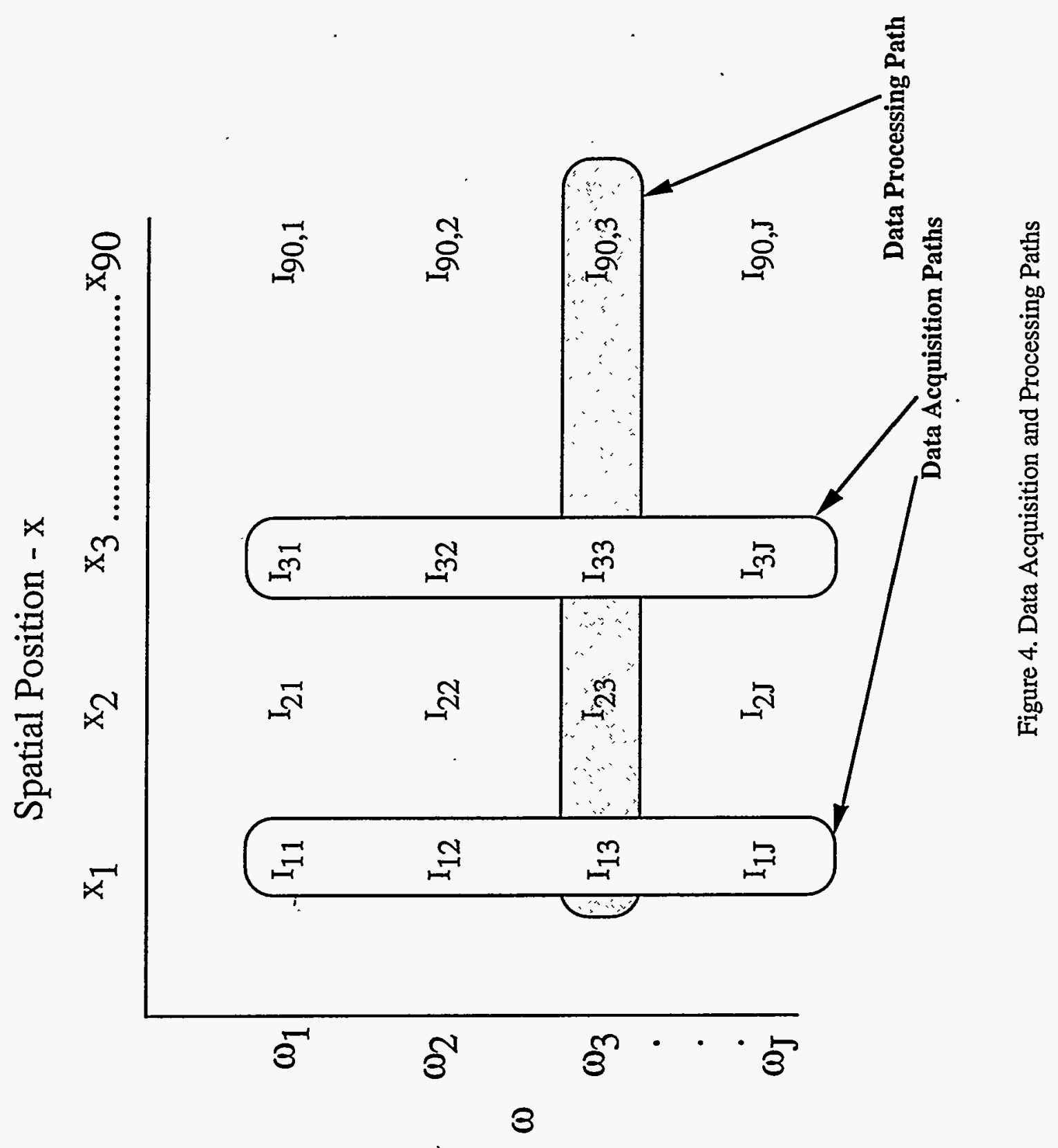


precision in the height of the wire above the ground plane, is unknown. All these uncertainties could be corrected with some further work in which numerical testing is offered as a potential fix to this dilemma.

\section{Experiment and Data}

\subsection{The Acquired Data}

An example of the data, as acquired, is shown in Figure 5 for two points along the wire, namely, Point 1 (the reference) and Point 20. For fixed position, the data represent the spectrum of the current and the plot is an example of the vertical strip in Figure 4. During the acquisition phase, ninety such data records were generated, i.e., current as a function of frequency for fixed position. In order to remove the source strength from the data, the current magnitude was normalized to the reference current at Point 1 and such a result is shown in Figure 6. The poor data behavior beyond $250 \mathrm{MHz}$ is due in large part to the signal level at Point 20 having fallen below the noise floor in the measurement system. In the vicinity of this anomalous behavior, the current at Point 20 was more than $60 \mathrm{~dB}$ below the current at Point 1 . And, in this frequency range, the current at Point 1 had already fallen more than an order of magnitude below its value at low frequencies. These combined effects could easily drive the signal below the system measuring capabilities. Also, there are instances where the current at Point 20 exceeds that at Point 1 . This can be due to uncertainties in the measurements or the presence of a standing wave on the structure.'

For the purposes of data analysis, the records were processed to provide an additional set of records which portray the current as function of position for fixed frequency. This presentation is representative of the horizontal strip in Figure 4 and a visual example of the data for such a "strip" is shown in Figure 7. The data in Figure 7 exhibit a substantial amount of variation on a point to point basis thus leading to questions regarding the source of this structure in the current distribution. Observations were made that the peaks did not move with changes in frequency, thus ruling out a strong standing wave on the line as the cause for these peaks. An investigation was made of the possibility that perturbations and inclusions in the concrete in the vicinity of the peaks might cause the excursions. Visual inspection of the pad and a study of photographs of the pad with the wire in place did not provide an obvious clue as to the cause. The possibility of rebar-induced perturbations in 


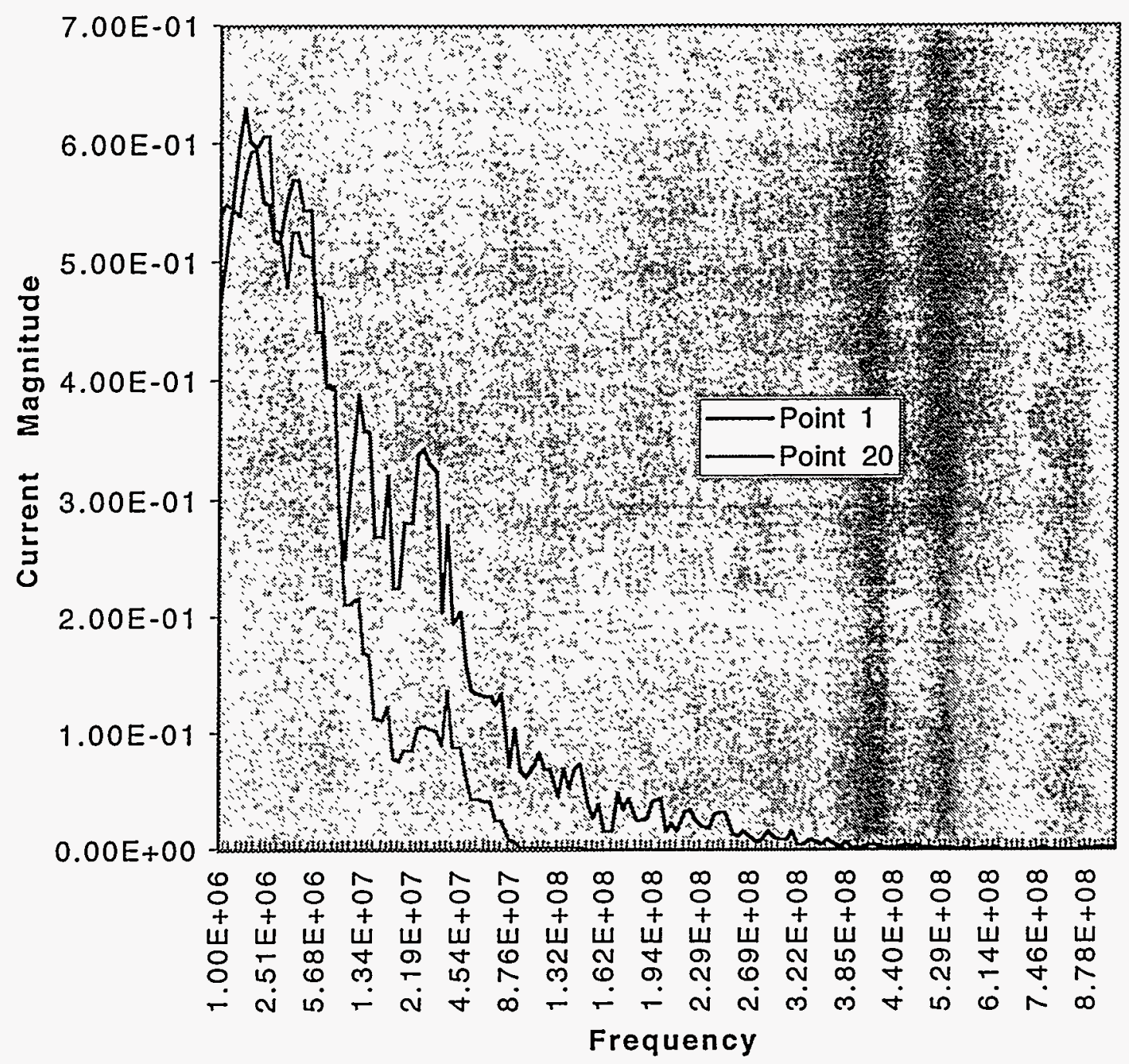

Figure 5. Current magnitude spectrum at Points 1 and 20 


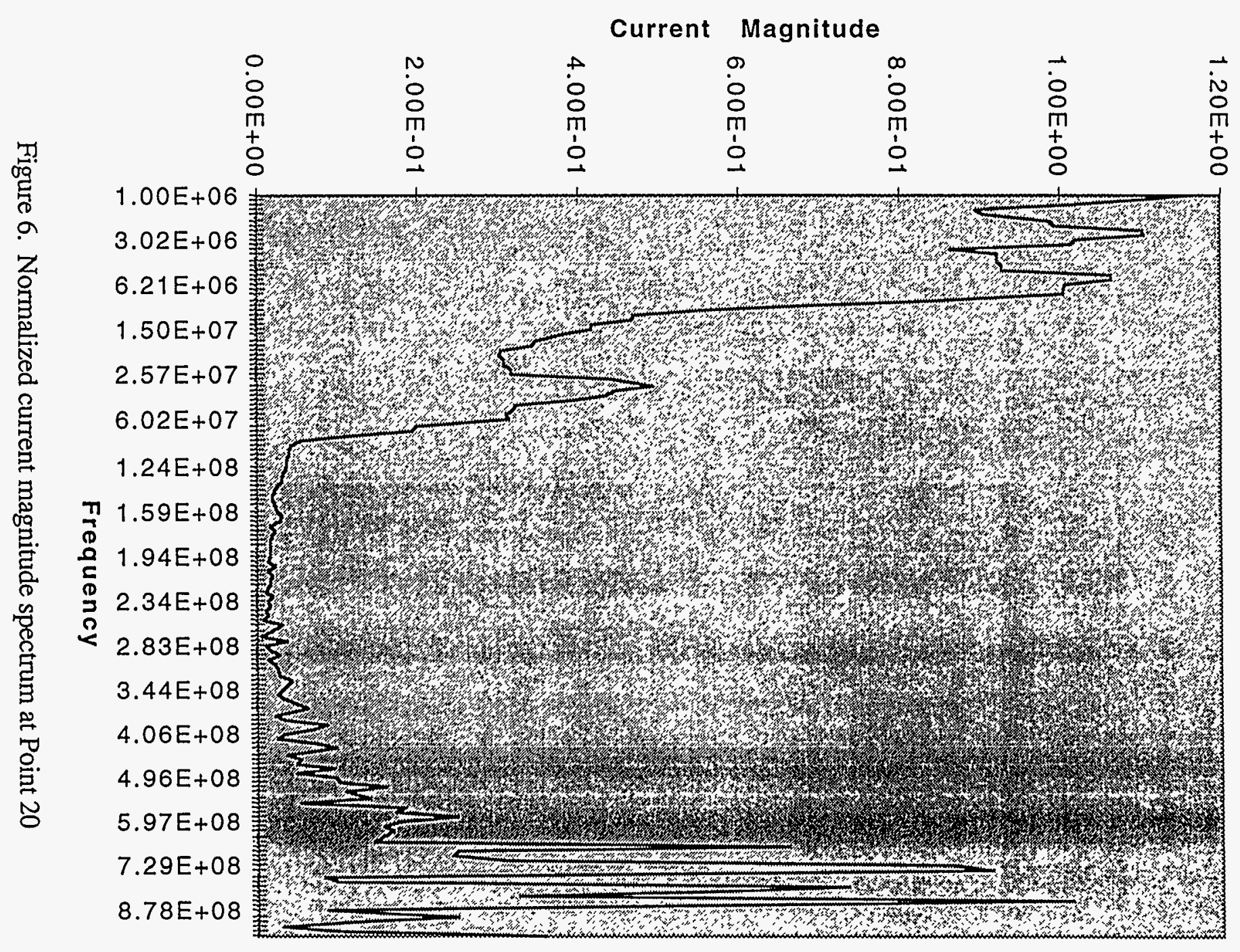




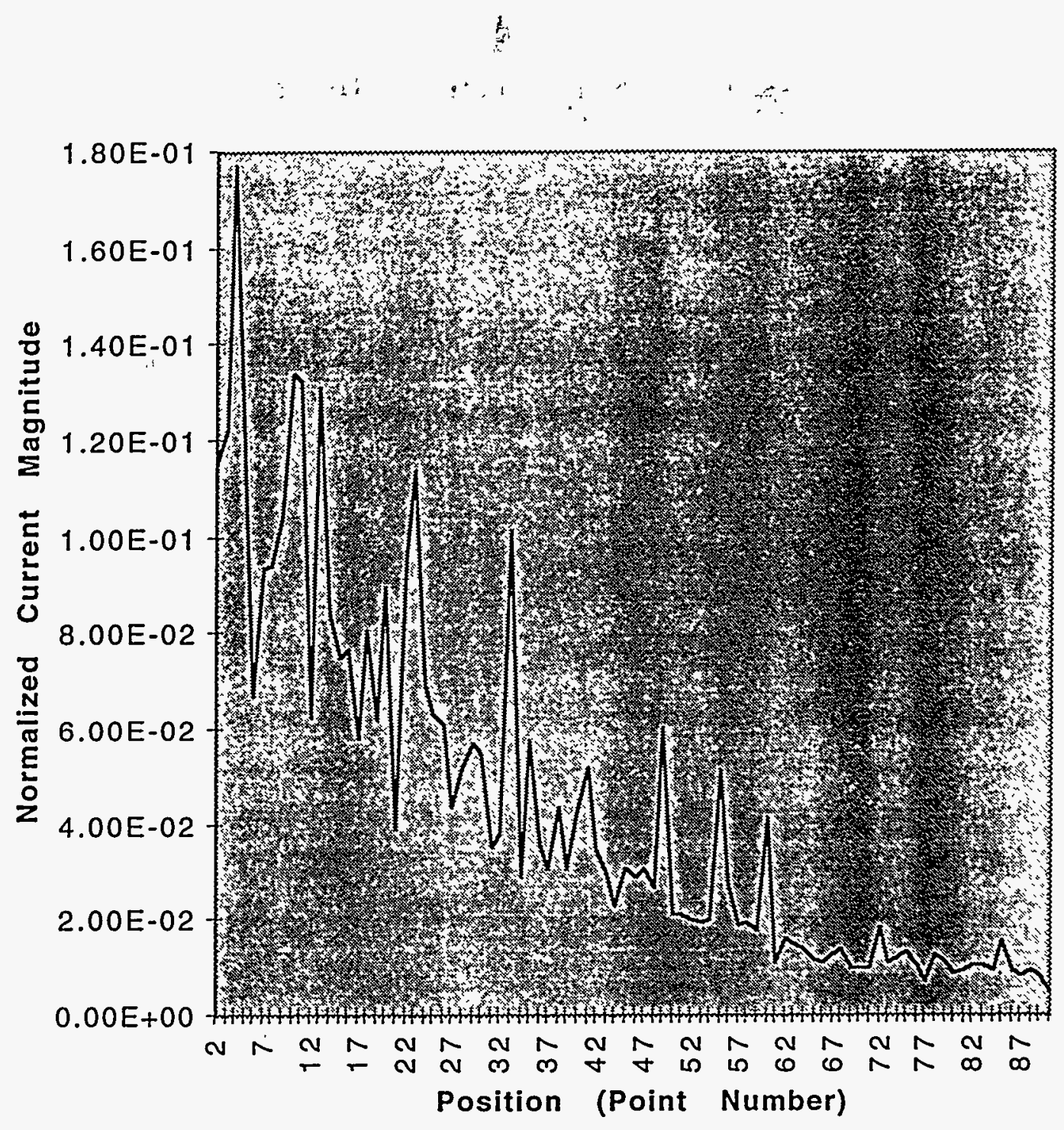

Figure 7 Normalized current as a function of position along the wire at $30 \mathrm{MHz}$ 
the fields and currents was also offered. However, modeling was performed using NEC [6] to analyze an end-driven wire very close ( $h=5 / 8$ inches) to a ground $\left(\varepsilon_{\mathrm{r}}=6, \sigma=0.006\right.$ $\mathrm{S} / \mathrm{m}$ ) with a single strand of perfectly conducting rebar that was buried at six inches. The effects on the wire current distribution were negligible except when the wire and rebar were parallel and aligned in the vertical plane. In the parallel case, the current showed perturbations in the average values of $\alpha$ and $\beta$ but nothing in its behavior as localized as seen in the measured data. Nothing could be detected which was capable of causing such a rapid change in current. Also, the pad was constructed of concrete squares and the wire was oriented diagonally across the squares. Given common concrete building practices, the rebar was most likely oriented parallel to the sides of the squares and orthogonal to edges so that it is unlikely that the rebar and wire were parallel. Other possibilities were considered, including measuring equipment malfunction.

A 3-D view of the data in the 10 to $275 \mathrm{MHz}$ range over the sampled extent of the wire allows a visualization of the previous discussions and is shown in Figure 8. The spectral behavior of the current (without normalization), its spatial behavior and its decay rates in these two variables is evident. The presence of the "spikes" or peaks in the current as a function of position mentioned earlier is also evident. Note that the "spikes" occur at identical positions independent of frequency and manifest themselves in this figure as ridges or striations at a constant position coordinate. Since the data acquisition for a single run was effected at a fixed position with frequency stepped, an offset or malfunction throughout a run could manifest itself as a frequency independent perturbation at a fixed location. A few sources of such error were considered. A malfunctioning or improperly placed or configured current probe could produce such perturbations. The current probes were of the "clothespin" type, that is, they were spring-loaded clamps that fit around the wire. These were repositioned for each run with only the reference at Point 1 unperturbed during the data acquisition process. Another possibility was inadvertent or improperly applied attenuators in the fiber optic link. ${ }^{1}$ Such a situation could be realized if the stepping attenuator system which was electronically controlled was sporadically "sticking" and reporting an incorrect value to the data acquisition system. At the present time, the identification of the source of the variability of the data has not been successful.

1 J. H. Millard, Private Communication 


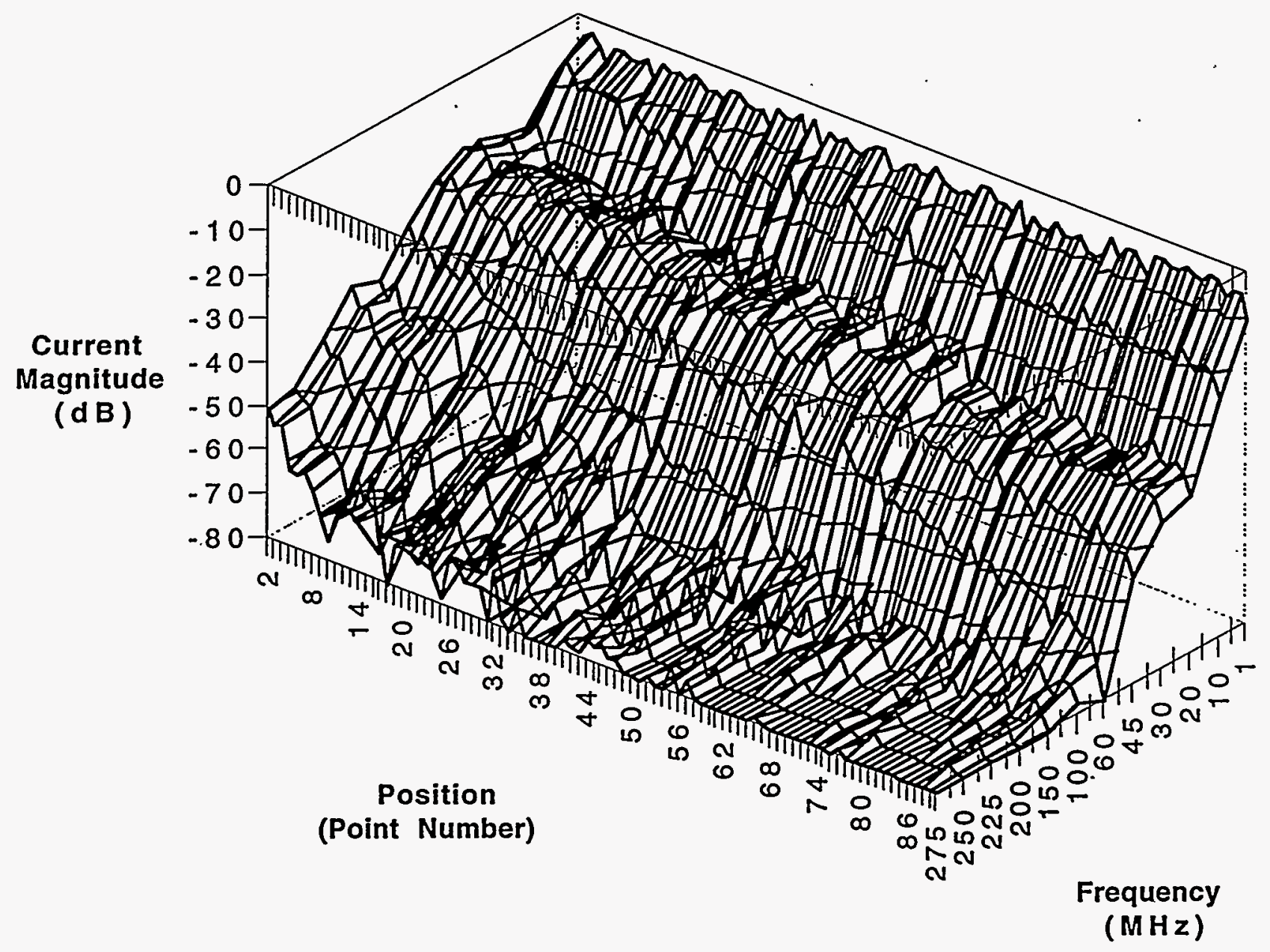

Figure 8. Magnitude of current on the wire as a function of position and frequency 


\subsection{Extraction of Attenuation and Phase Constants}

As explained in Section 2.1, the component of current of diagnostic value is the transmission line mode which is dominant in a window from the source to a point where it drops below the branch cut mode. Hence, we need to locate this region on the wire and estimate the attenuation and phase constants. Given that the current can be represented by

$$
\mathrm{I}(\mathrm{x}, \omega)=\mathrm{I}_{0} \exp \left[\mathrm{i}(\beta(\omega)+\mathrm{i} \alpha(\omega))\left(\mathrm{x}-\mathrm{x}_{1}\right)\right], \mathrm{k} \gamma=\beta+\mathrm{i} \alpha
$$

an exponential fit to the acquired data (or a normalized version of the data) or a linear fit to the log of the data can provide values for the normalized attenuation and phase constants. Using the complex natural logarithm of the data, one has

$$
-[\alpha(\omega)-i \beta(\omega)]\left(x-x_{1}\right)+\operatorname{Ln}\left(I_{0}\right)=\operatorname{Ln}(I(x, \omega))
$$

so that, for two points along the structure at $x_{i}$ and $x_{j}$ at a fixed frequency $\omega_{n}$, one can solve for $\alpha\left(\omega_{n}\right)-i \beta\left(\omega_{n}\right)$

$$
\alpha\left(\omega_{n}\right)-i \beta\left(\omega_{n}\right)=\left[\operatorname{Ln}\left(I\left(x_{i}, \omega_{n}\right)\right)-\operatorname{Ln}\left(I\left(x_{j}, \omega_{n}\right)\right)\right] /\left(x_{j}-x_{i}\right)
$$

Keep in mind that these are complex natural logs. Using the real and imaginary parts of the equation with the current represented by II $(\mathrm{x}, \omega) \operatorname{lexp}(\mathrm{j} \phi(\mathrm{x}, \omega))$, with $\phi(\mathrm{x}, \omega)$ the phase at $\mathrm{x}$ and $\omega$, it is a simple matter to extract $\alpha\left(\omega_{n}\right)$ and $\beta\left(\omega_{n}\right)$ and present them in common log notation thus allowing easy conversion to $\mathrm{a} \mathrm{dB}$ scale

$$
\begin{aligned}
& \alpha\left(\omega_{n}\right)=2.3025\left[\log \left|I\left(x_{i}, \omega_{n}\right)\right|-\log \mid I\left(x_{j}, \omega_{n}\right)\right] /\left(x_{i}-x_{j}\right) \\
& \beta\left(\omega_{v}\right)=\left[\phi\left(x_{i}, \omega_{n}\right)-\phi\left(x_{j}, \omega_{n}\right)\right] /\left(x_{i}-x_{j}\right)
\end{aligned}
$$

In the face of the variability of the data, a Least Squares (LSQ) fit to the data was performed. A linear fit was performed to the log magnitude and phase of the data, individually. Also, a complex exponential was LSQ fit to the complex data. The differences were minimal and the following relates to separate fits to the magnitude and phase information as represented in Equations (9) and (10).

The difficulty in performing LSQ fits to the data for the wire above ground arises from two sources: 1) the measurement system had its limitations and beyond a certain spatial point the data could become "noisy" as the noise floor was encountered and 2) contributions to the current other than the transmission line mode could become dominant at sufficiently large distances at high frequencies. Since the branch cut term decays as $\mathrm{x}^{-2}$, it always prevails over the transmission line mode at sufficiently large distances and manifests itself as a flattening of the current magnitude distribution with distance from the source. In fact, 
modeling of the physical configuration under consideration has confirmed such characteristics. But, the noise floor could provide a similar behavior. Since it does not matter that we identify the structure's cause but only avoid it in our LSQ fits, the initial data fitting involved some "eyeballing" of the data. This required inspection of the log(current magnitude) and of the phase to perform a fit to that part which looked "roughly linear". The danger here is that small errors in the resulting value of attenuation and phase constant could ultimately lead to large errors in the $\varepsilon_{\mathrm{r}}$ and $\sigma$ if the method of solution was not robust.

Examples of the process of evaluating $\alpha$ and $\beta$ are illustrated in Figures 9 through 12. In Figures 9 and 10 the magnitude and phase data for $30 \mathrm{MHz}$ are shown. Also shown in the Figures 9 and 10 are the linear fits to the log magnitude and phase over the entire range (including all points from 2 through 90), respectively. Figures 11 and 12 show the respective plots for a frequency of $100 \mathrm{MHz}$. There, the magnitude and phase deviate from linear beyond certain points along the wire. Over the linear portions in both cases one can see the rapidly attenuating slow wave characteristic of the transmission line mode. Of course, when the magnitude begins to decay at a slower rate we may be seeing either the surface attached mode, the branch cut contribution, or evidence of the noise floor. When the phase deviates from linear and slow wave, we begin to see a wave that is increasing in velocity' and may eventually reach the velocity in the upper medium or become the fast wave whose existence is predicted theoretically. The orderly behavior of the phase suggests that the cause of the flattened current distribution is not the noise floor but rather the branch cut . Ultimately, however, this issue does not impact our analysis as it is the transmission line mode that provides attenuation and phase constants that are most important in the evaluation of the relative dielectric constant and conductivity.

\subsection{Determination of Relative Dielectric Constant and Conductivity}

The process of solving for $\varepsilon_{\mathrm{r}}$ and $\sigma$ has already been touched upon. Once values for $\alpha$ and $\beta$ in the transmission line mode are obtained for a given frequency, the value of $\gamma_{p}=\beta+i \alpha$ is determined. An iterative solution of the equation $M\left(\gamma_{p}\right)=0$ for $n$, the index of refraction for the medium, yields $\varepsilon_{\mathrm{r}}$ and $\sigma$ since $n=\left(\varepsilon_{\mathrm{r}}+\mathrm{i} \sigma /\left(\omega \varepsilon_{\mathrm{p}}\right)\right)^{1 / 2}$. The values of $\varepsilon_{\mathrm{r}}$ and $\sigma$ can range from 1 and 0 , respectively, to infinity, although real ground will have a much more limited range. For $\varepsilon_{\mathrm{r}}=1$ and $\sigma=0$, the values of $\alpha$ and $\beta$ will be nearly $\alpha=0$ and $\beta=k$. 


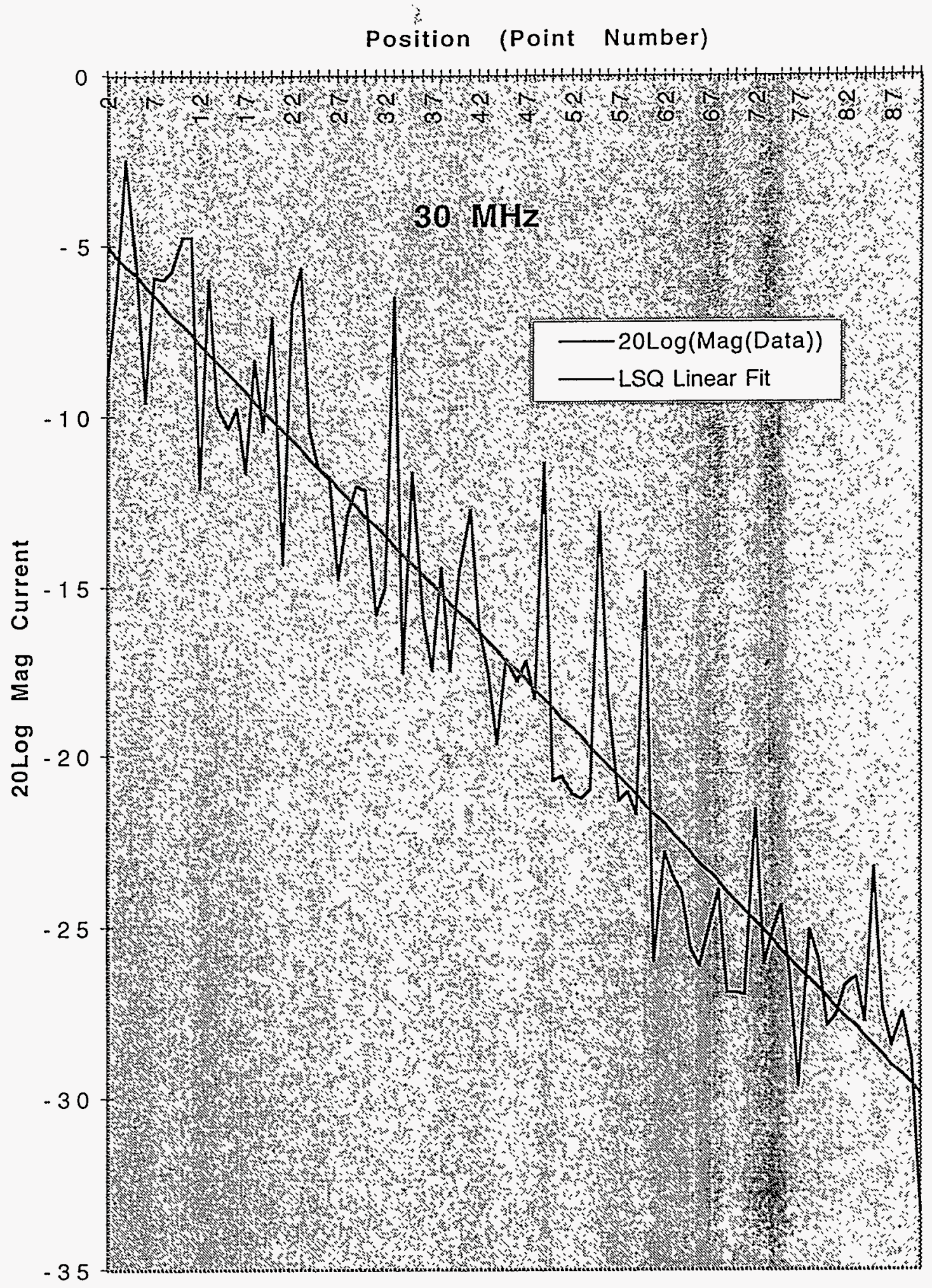

Figure 9. Log magnitude of normalized current and linear LSQ fit over Points 2 to 90 


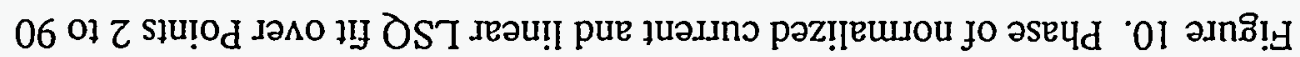

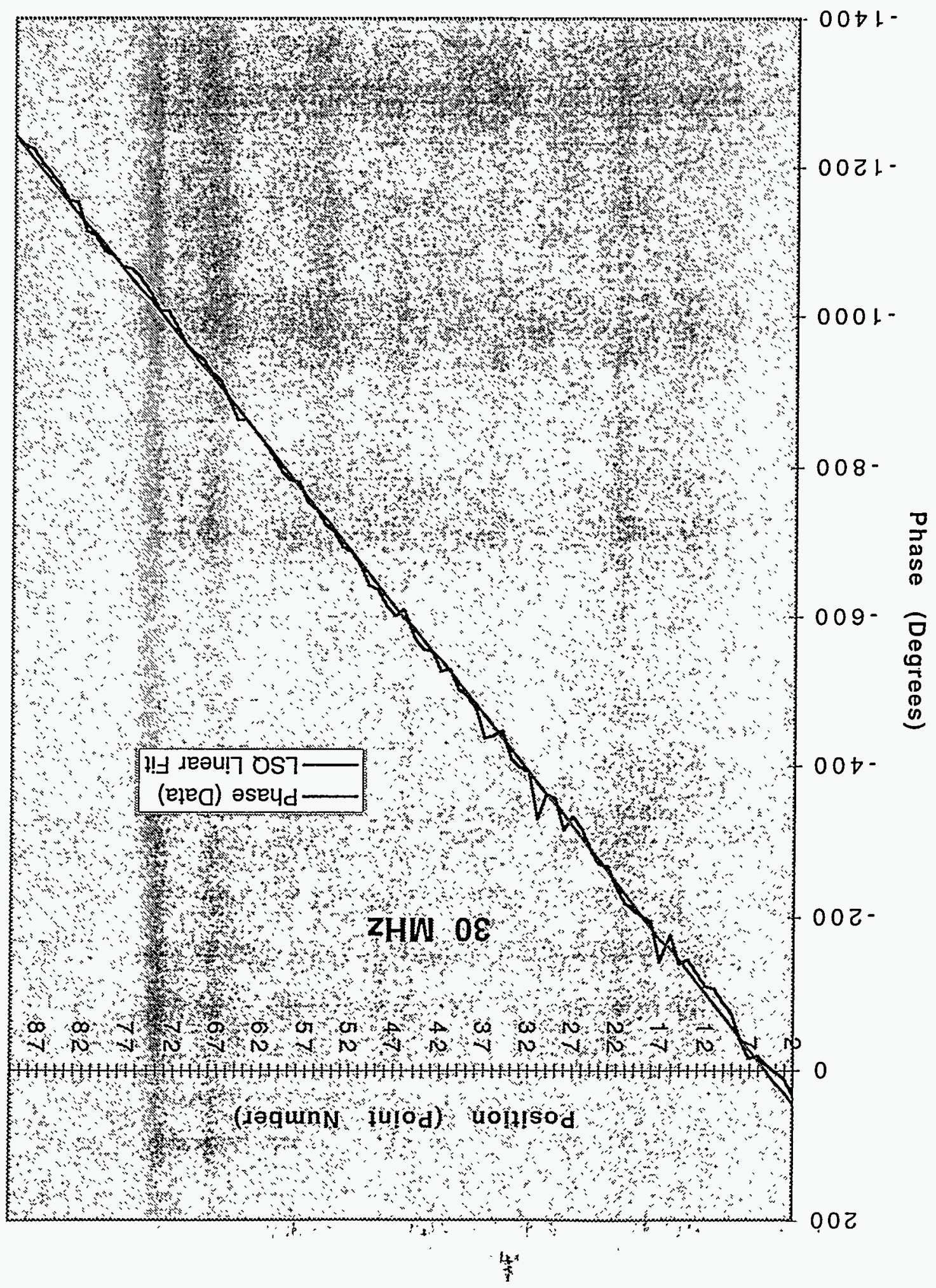




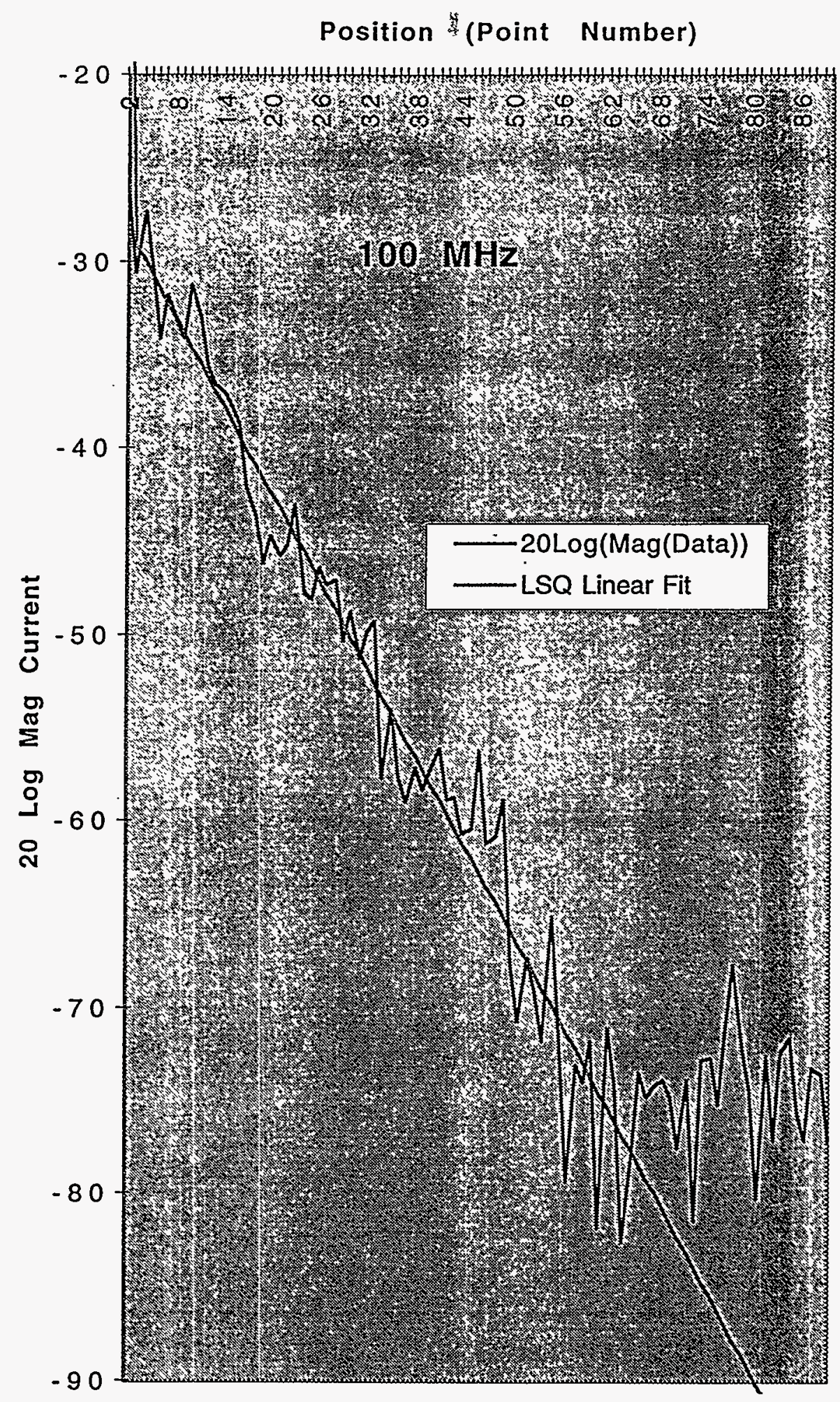

Figure 11. Log magnitude of normalized current and linear LSQ fit over Points 2 to 66 


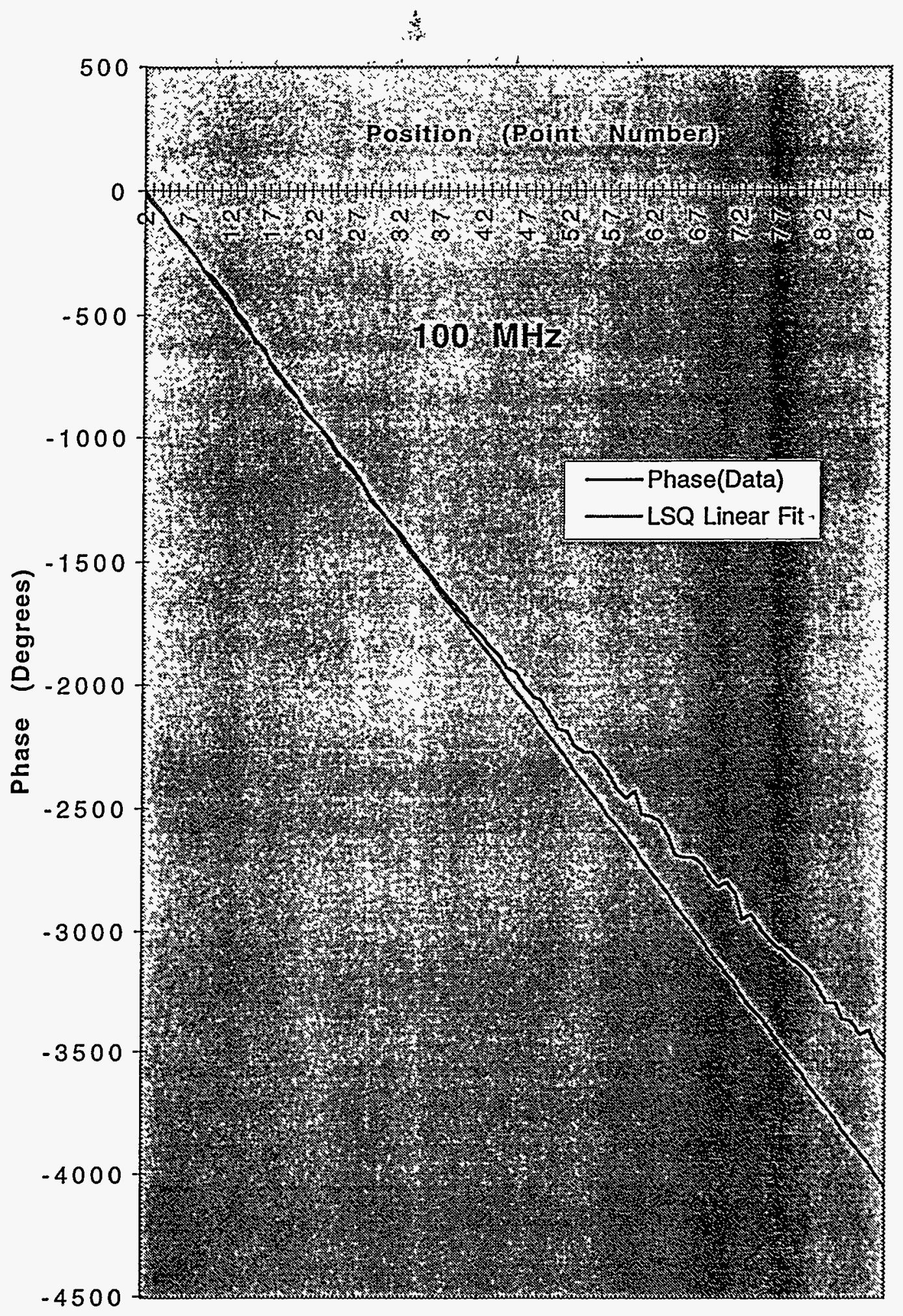

Figure 12. Phase of normalized current and linear LSQ fit over Points 2 to 30 


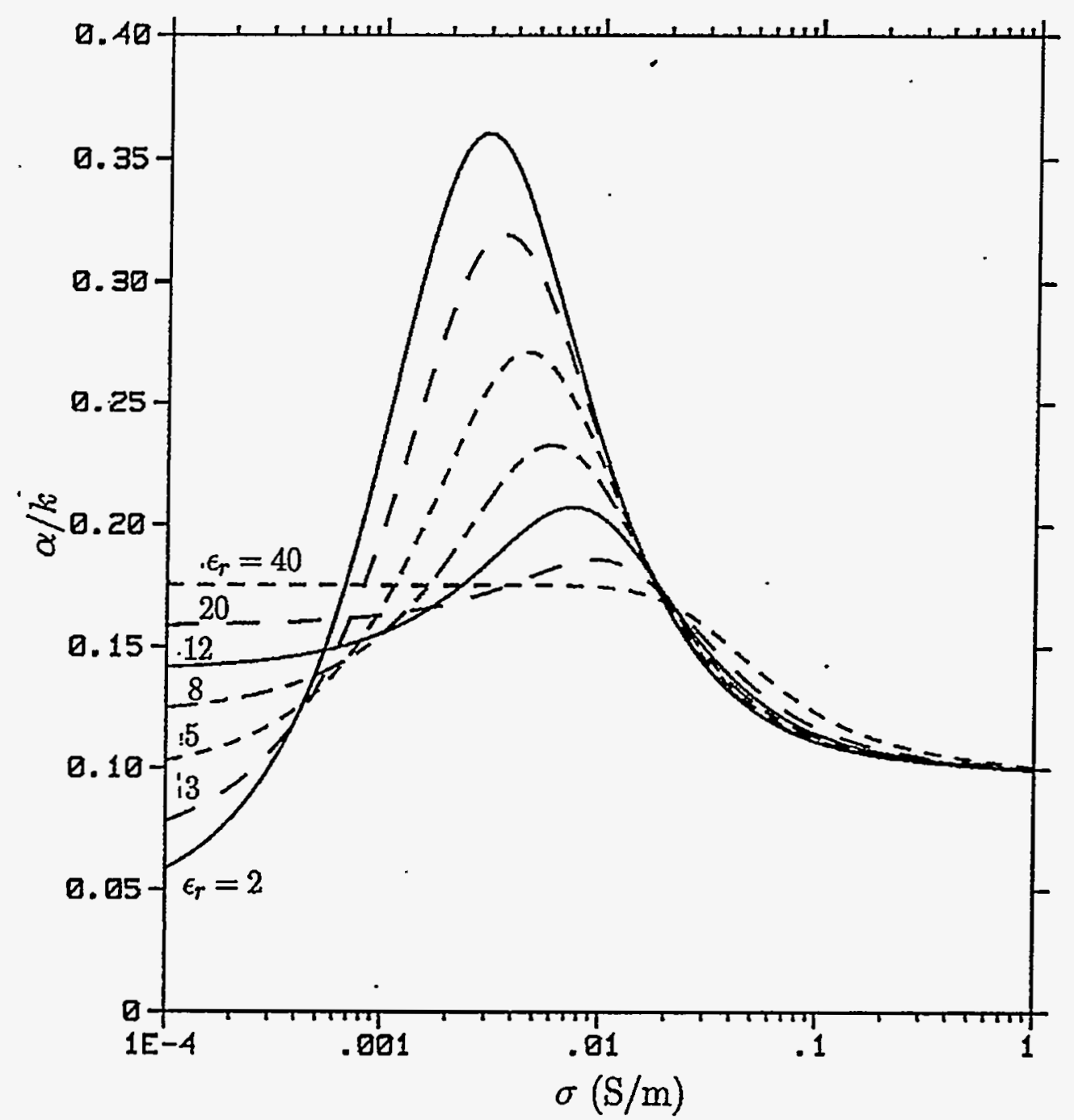

Figure 13. Normalized attenuation constant vs. conductivity for various $\varepsilon_{\mathrm{r}}$ at $10 \mathrm{MHz}$ 


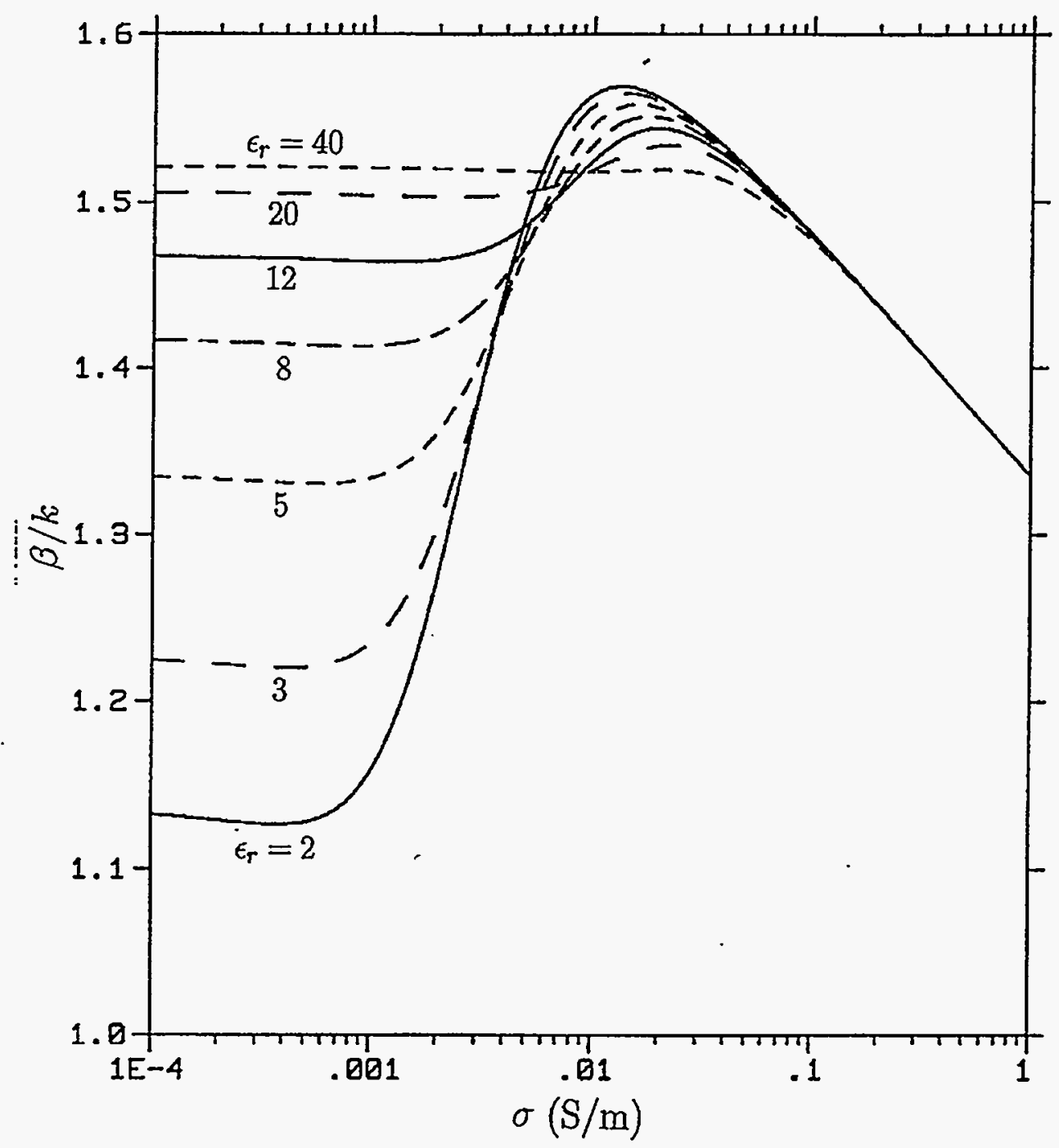

Figure 14. Normalized phase constant vs. conductivity for various $\varepsilon_{\mathrm{r}}$ at $10 \mathrm{MHz}$ 
the other hand, the behavior of the log magnitude of the current at $250 \mathrm{MHz}$ which is shown in Figure 15 leads to much greater difficulty in defining the linear region and thus leads to greater uncertainty in establishing the value for the attenuation constants. The phase constant determination is also difficult at high frequencies. Figure 16 is the phase plot over 17 points on the wire at $250 \mathrm{MHz}$. Clearly, the phase change per cell is quite large and errors are possible. Given the available data and the inability to redo portions of the experiment, a range was determined over which the uncertainties in the values for $\alpha$ and $\beta$ was considered small. For the purposes of our analysis this range was defined as approximately $5 \mathrm{MHz}<\mathrm{f}<150 \mathrm{MHz}$. The lower limit was also consistent with concerns of the experiment personnel regarding their ability to make accurate measurements on the pad in view of previous experience with adverse ground loops and other low frequency limitations. Figure 17 and 18 represent the normalized constants ( $\alpha / k$ and $\beta / \mathrm{k}$ ) versus frequency as determined from the experimental data.

The data for $\alpha$ and $\beta$ were used to determine the effective relative dielectric constant and the conductivity of the half space beneath the wire. The process was reasonably robust in the area defined for confidence in estimates of attenuation and phase constants. Outside this range, the process might not return a solution ( values of $\varepsilon$ and $\sigma$ were not available which would simultaneously satisfy $M\left(\gamma_{p}\right)=0$ ). In some cases, "near" solutions were available in that a given value of $\varepsilon_{\mathrm{r}}$ would not yield a unique value of $\sigma$ but $M(\gamma)$ was almost equal to zero for a range of $\sigma$. These data were not used in this analysis.

\section{Analysis}

In order to extend our estimates for $\varepsilon_{\mathrm{r}}$ and $\sigma$ beyond the range where reliable values of $\alpha$ and $\beta$ were determined, a theoretical model was used as the underpinning for the results. Such models have been developed in the literature and, for orientational-polarization effects, one has the Debye equations $[7,8]$

$$
\begin{aligned}
& \varepsilon_{\mathrm{r}}=\varepsilon_{\text {rinf }}+\left(\varepsilon_{\mathrm{rs}}-\varepsilon_{\mathrm{rs}}\right) /\left(1+\omega^{2} \tau^{2}\right) \\
& \sigma=\omega \tau\left(\varepsilon_{\mathrm{rs}}-\varepsilon_{\mathrm{rs}}\right) /\left(1+\omega^{2} \tau^{2}\right)
\end{aligned}
$$

where $\varepsilon_{\text {inf }}$ is the optical limit for the permittivity, $\varepsilon_{\mathrm{rs}}$ is the dc value, and $\tau$ is the orientational relaxation time for this model. Plots versus frequency of $\varepsilon_{\mathrm{r}}$ and $\sigma$ extracted from the 


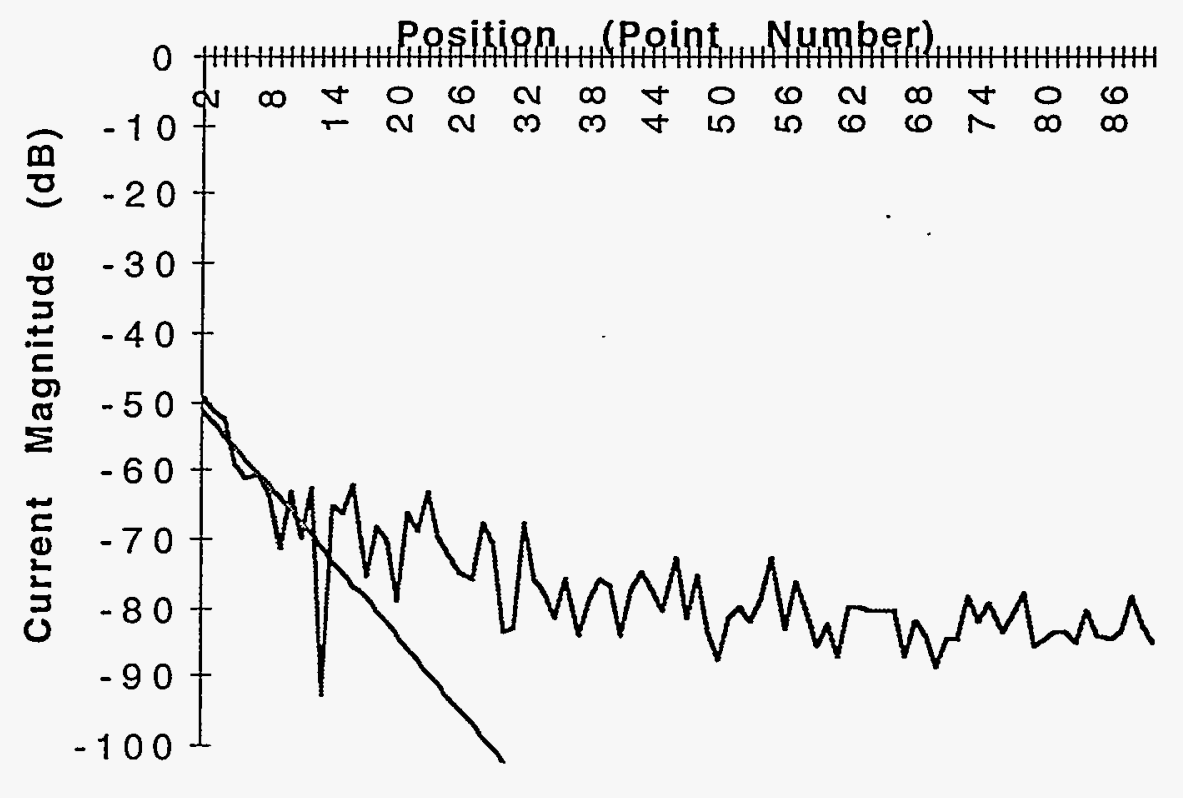

Figure 15. Current Magnitude in $\mathrm{dB}$ and LSQ fit at $250 \mathrm{MHz}$

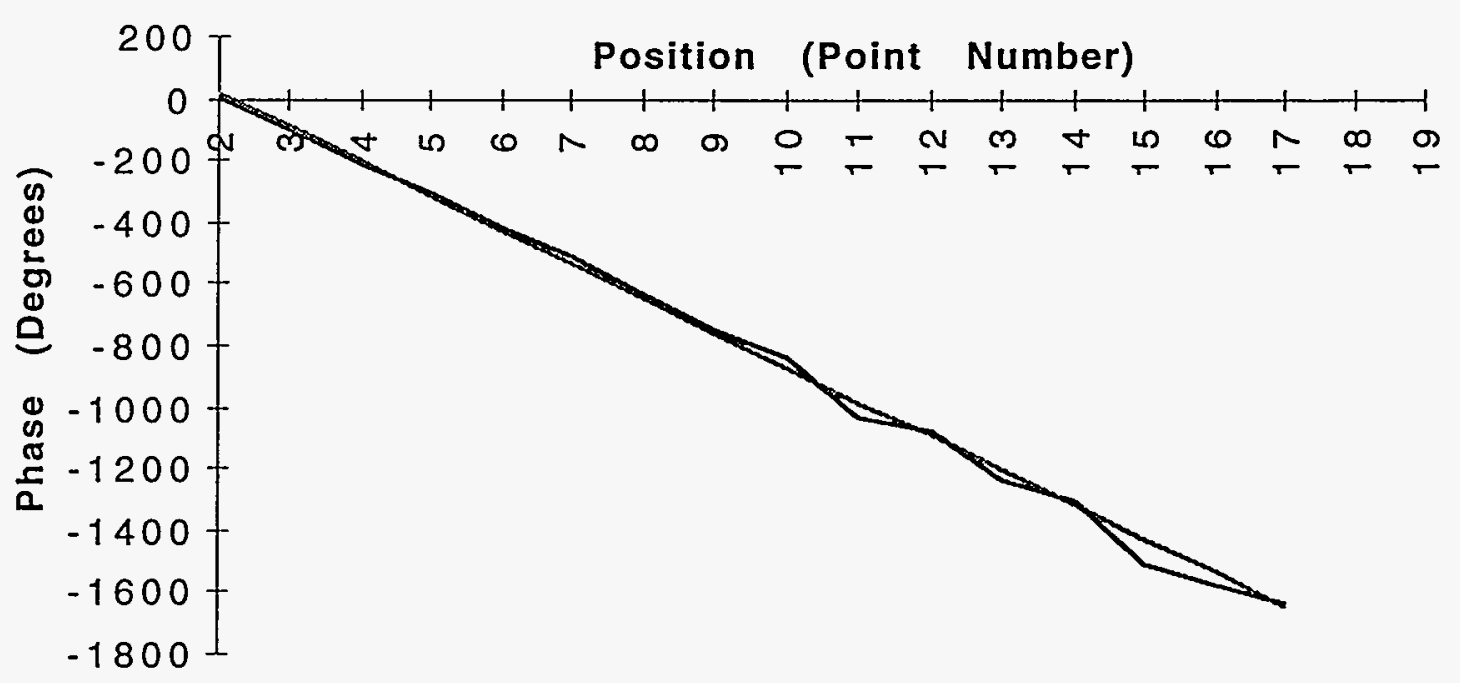

Figure 16. Phase of current on wire and LSQ fit over 17 points at $250 \mathrm{MHz}$ 


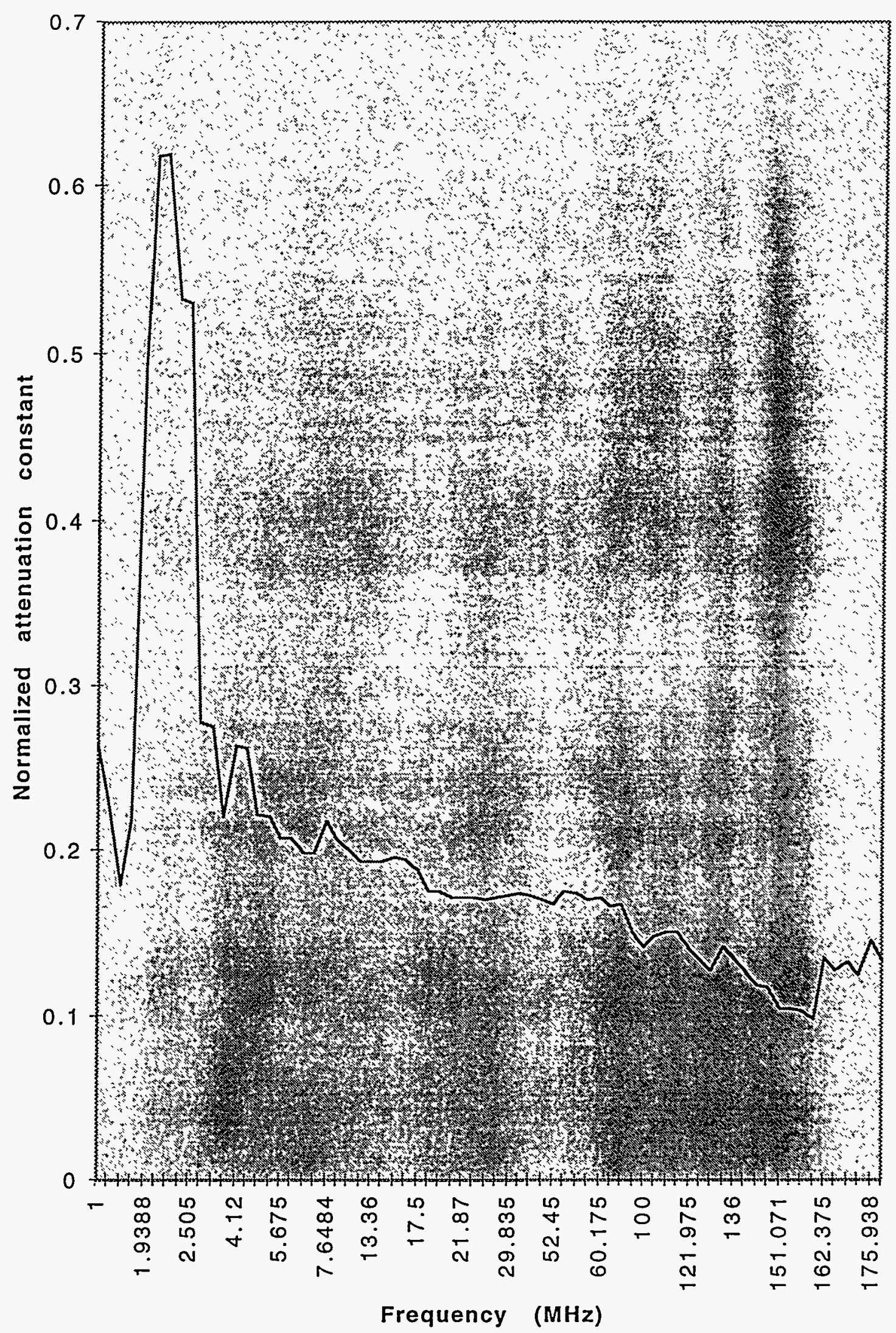

Figure 17. Normalized attenuation constant versus frequency 


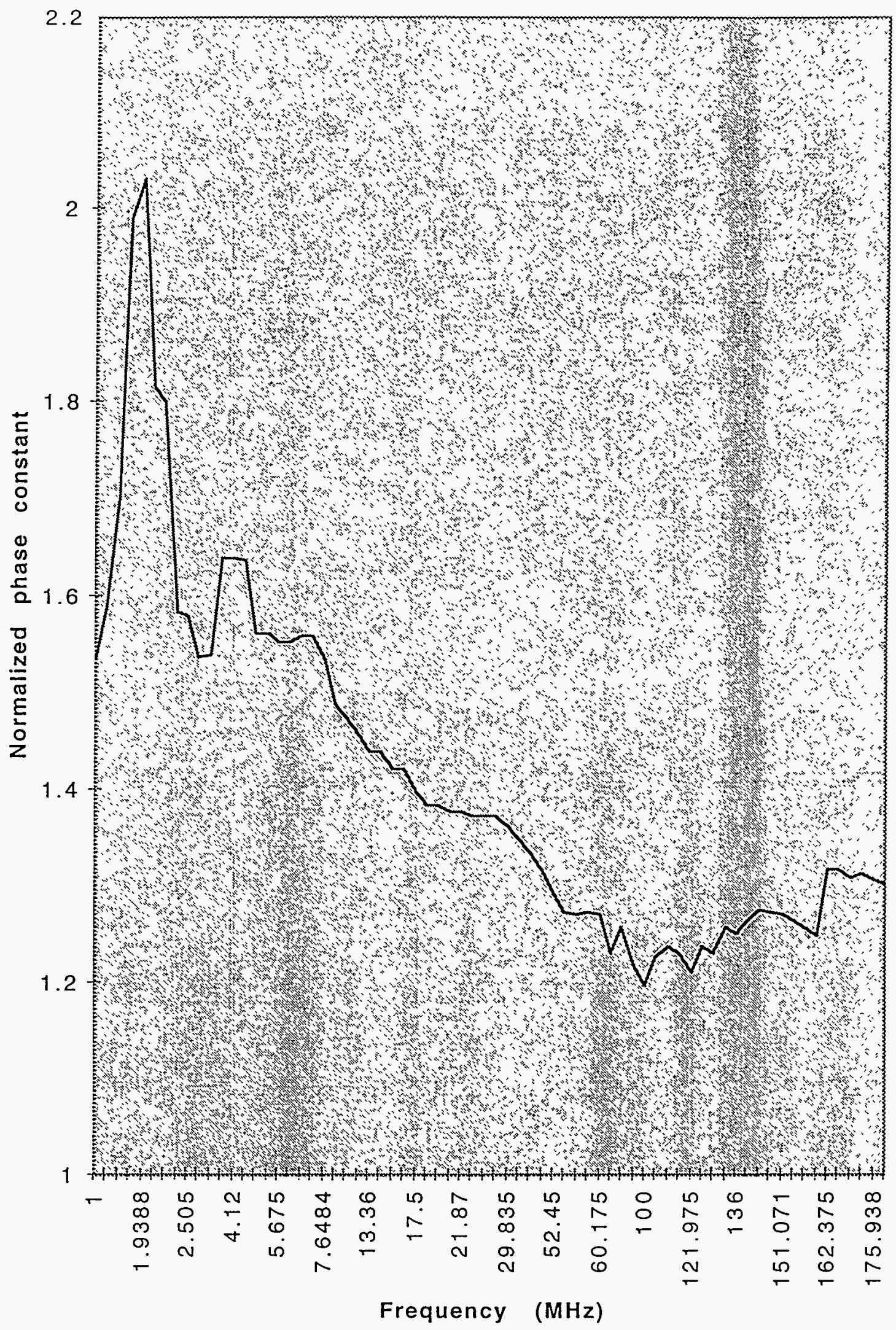

Figure 18. Normalized phase constant versus frequency 
measured current are shown in Figures 19 and 20 , respectively. The anomalous behavior of $\sigma$ in the range 60 to $80 \mathrm{MHz}$ is not well understood. Also shown in each figure is a least squares fit to the model for the behavior of the constitutive parameters versus frequency. In the LSQ fits performed for the data in hand, the values of $\varepsilon_{\mathrm{rs}}, \varepsilon_{\text {inf }}$ and $\tau$ were not the same for the fits of the two sets of data. Clearly, such agreement for the two models would be expected for a perfect model as would $\varepsilon_{\mathrm{r}}$ and $\sigma$ pairs in agreement with the Kronig-Kramers relationships [9]. For the curves shown, however,

$$
\begin{aligned}
& \varepsilon_{\mathrm{r}}=4.2418+6.1943 /\left(1+1.129 \cdot 10^{-17} \cdot \omega^{2}\right) \\
& \sigma=4.416 \cdot 10^{-11} \cdot \omega /\left(1+1.354 \cdot 10^{-17} \cdot \omega^{2}\right) \text { for the full range of data } \\
& \sigma=3.6385 \cdot 10^{-11} \cdot \omega /\left(1+1.135 \cdot 10^{-17} \cdot \omega^{2}\right) \text { for the restricted data }
\end{aligned}
$$

The restricted data set is composed of the full range of data with frequencies below $18 \mathrm{MHz}$ excluded. The behavior of the permittivity over the frequency range shown is orderly and constrained while that of the conductivity is more variable. Also, the models seem to fit the behavior of the permittivity better than the conductivity. The impact of these issues on propagation in the upper half space is adequately reflected in the variation of the reflection coefficients with variations in the lower medium's wavenumber (or index of refraction, $n$ ) which depends on $\varepsilon_{\mathrm{r}}$ and $\sigma$. This wavenumber arises repeatedly in the analyses and is a key determinant of the reflected and transmitted waves at the interface of the materials when a plane wave, for instance, is incident. The wavenumbers in the pad medium and free space, respectively, are given by

$$
\begin{aligned}
& \mathrm{k}_{1}=\mathrm{k}_{0} \mathrm{n}=\mathrm{k}_{0}\left[\varepsilon_{\mathrm{r}}\left(1+\mathrm{i} \sigma /\left(\omega \varepsilon_{0} \varepsilon_{\mathrm{r}}\right)\right]^{1 / 2}\right. \\
& \mathrm{k}=\mathrm{k}_{0}=\omega\left[\mu_{0} \varepsilon_{0}\right]^{1 / 2}
\end{aligned}
$$

Two limiting types of propagation in the lower half space are possible, i.e., the conditions under which propagation is conduction or displacement current dominated. Conduction current domination will occur when $\sigma \gg \omega \varepsilon_{0} \varepsilon_{\mathrm{r}}$ and will result in a propagation constant that is essentially independent of the relative dielectric constant in the ground, $\varepsilon_{\mathrm{r}}$. On the other hand, displacement current will dominate when $\sigma<<\omega \varepsilon_{0} \varepsilon_{\mathrm{r}}$ and will result in propagation characteristics relatively insensitive to conductivity, $\sigma$, and frequency, $\omega$. The "boundary" between these two regions can be said to be approximately

$$
\sigma=\omega \varepsilon_{0} \varepsilon_{\mathrm{r}}
$$

Figure 21 shows such boundaries for frequencies of $f=10,20,50,100,400$, and 1000 $\mathrm{MHz}$. Also shown in the figure is a contour connecting elements of the set of $\{\varepsilon, \sigma\}$ pairs that have been determined experimentally. The sequencing, which is in ascending values 


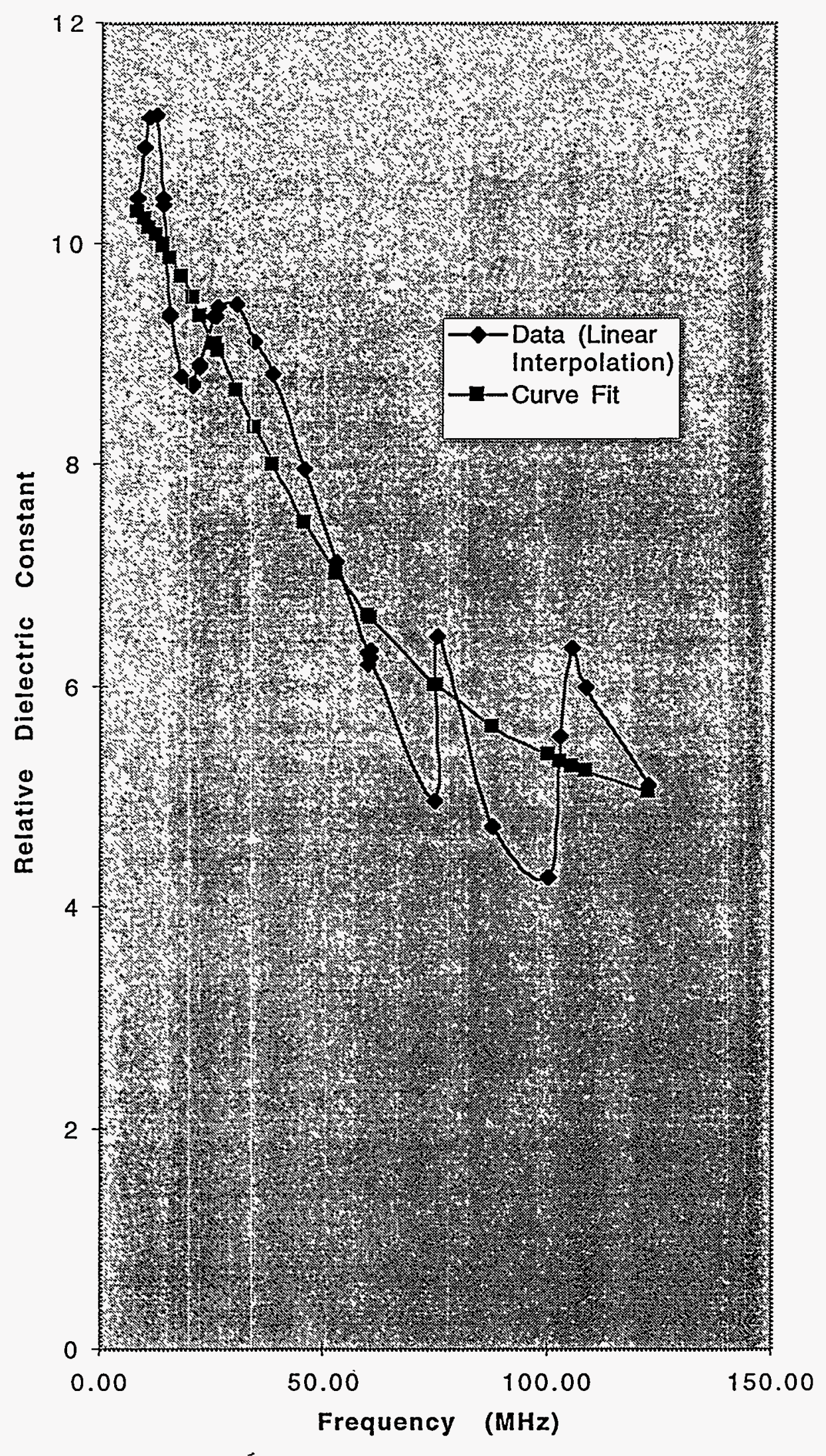

Figure 19. Relative dielectric constant versus frequency - experimental data and curve fit 


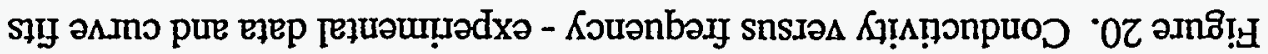

\section{(zHW) Kouənbədঊ}

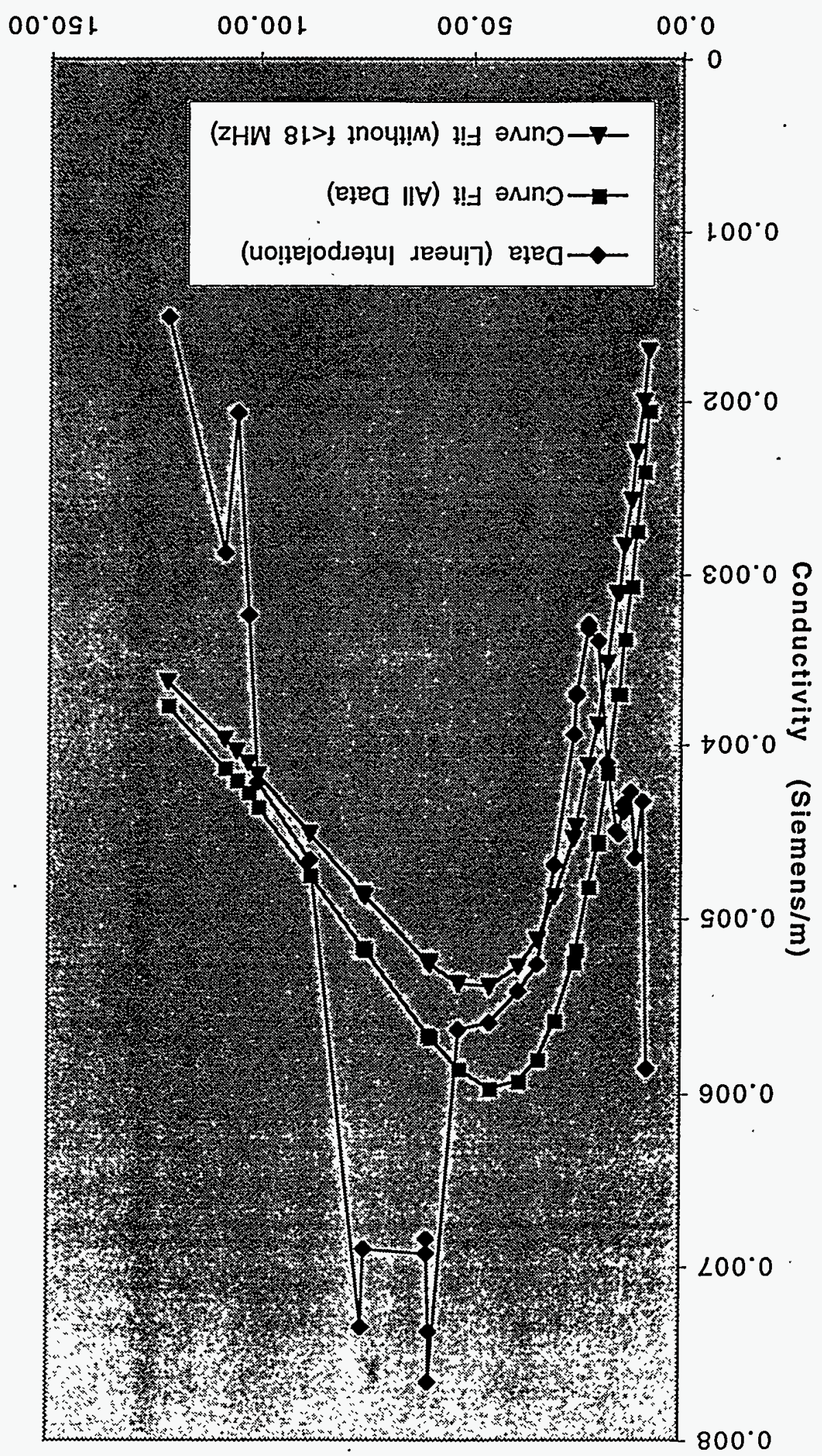




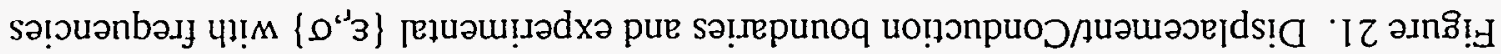

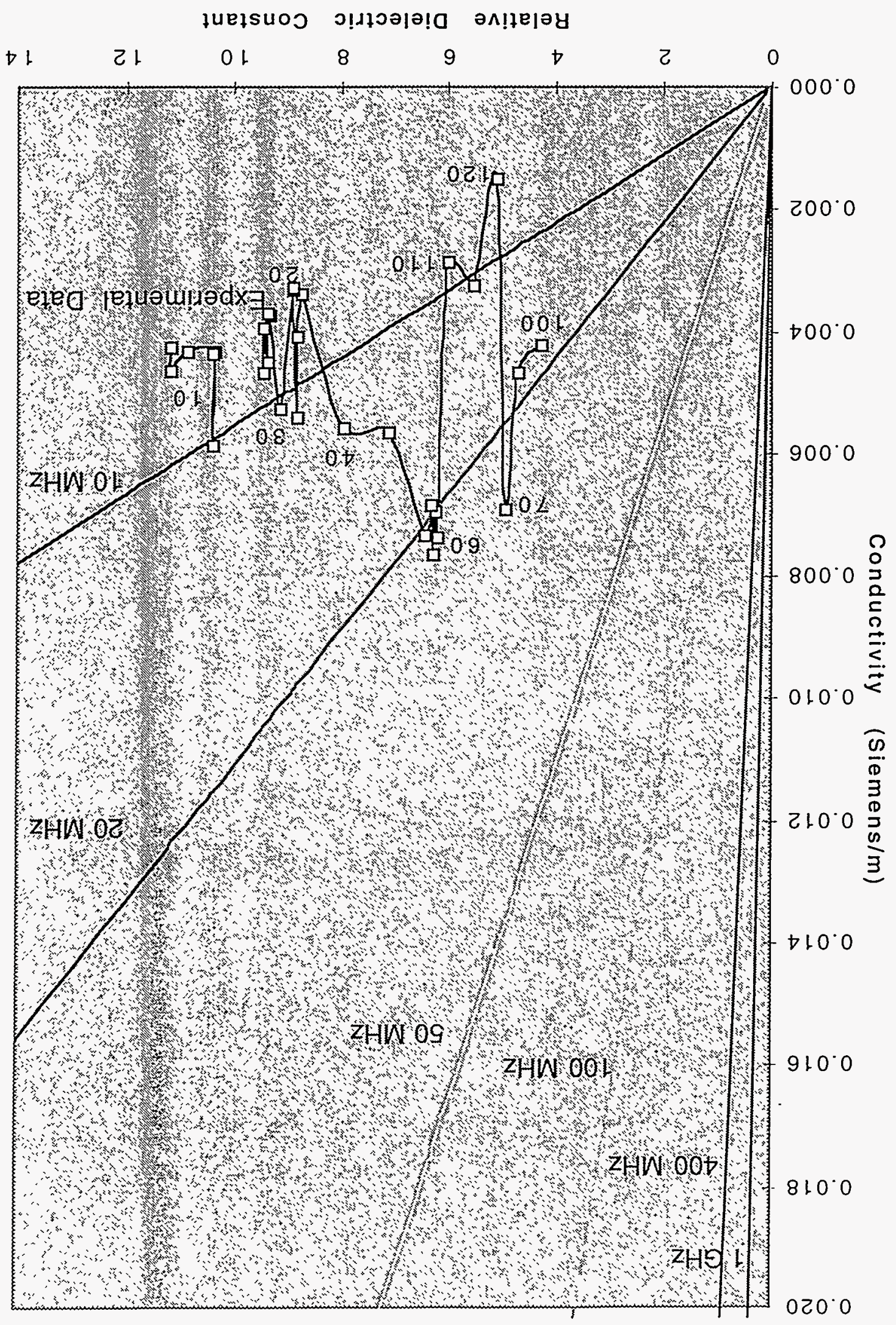


of conductivity, does not convey any physical information. The important feature to be observed in the figure is the range of the $\{\varepsilon, \sigma\}$ set and the fact that the propagation is displacement current dominated at frequencies above about 20 to $50 \mathrm{MHz}$. Another feature is that for the material under study, using average values over the frequency range $\left(f_{1}, f_{2}\right)$ of Equations (13) and (14),

$$
\begin{aligned}
& <\varepsilon_{\mathrm{r}}\left(\mathrm{f}_{1}, \mathrm{f}_{2}\right)>=4.24+2.93 \cdot 10^{8} \cdot\left[\tan ^{-1}\left(2.11 \cdot 10^{8} \cdot \mathrm{f}_{2}\right)-\tan ^{-1}\left(2.11 \cdot 10^{8} \cdot \mathrm{f}_{1}\right)\right] /\left(\mathrm{f}_{2}-\mathrm{f}_{1}\right) \\
& <\sigma\left(\mathrm{f}_{1}, \mathrm{f}_{2}\right)>=2.595 \cdot 10^{5} \cdot \ln \left[\left(1+5.29 \bullet 10^{-16} \cdot \mathrm{f}_{2}^{2}\right) /\left(1+5.29 \bullet 10^{-16} \bullet \mathrm{f}_{1}^{2}\right)\right] /\left(\mathrm{f}_{2}-\mathrm{f}_{1}\right)
\end{aligned}
$$

one finds that average values of $\varepsilon_{\mathrm{r}} \sim 7$ and $\sigma \sim 0.005$ appear to be reasonable over the 10 to $100 \mathrm{MHz}$ range. Of course, for frequencies above $50 \mathrm{MHz}$ the values of the conductivity may be of secondary importance.

To further support the observations based on the behavior of the propagation constant or wavenumber in the pad material, attention was directed towards the Fresnel reflection coefficients at the pad interface. These reflection coefficients are given by

$$
\begin{aligned}
& \mathrm{R}_{\mathrm{H}}=\left[\mu_{1} \mathrm{k}_{0} \cos \theta-\mu_{0}\left(\mathrm{k}_{1}^{2}-\mathrm{k}_{0}{ }^{2} \sin ^{2} \theta\right)^{1 / 2}\right] /\left[\mu_{1} \mathrm{k}_{0} \cos \theta+\mu_{0}\left(\mathrm{k}_{1}{ }^{2}-\mathrm{k}_{0}{ }^{2} \sin ^{2} \theta\right)^{1 / 2}\right] \\
& \mathrm{R}_{\mathrm{E}}=\left[\mu_{0} \mathrm{k}_{1}^{2} \cos \theta-\mu_{1} \mathrm{k}_{0}\left(\mathrm{k}_{1}{ }^{2}-\mathrm{k}_{0}{ }^{2} \sin ^{2} \theta\right)^{1 / 2}\right] /\left[\mu_{0} \mathrm{k}_{1}^{2} \cos \theta+\mu_{1} \mathrm{k}_{0}\left(\mathrm{k}_{1}{ }^{2}-\mathrm{k}_{0}{ }^{2} \sin ^{2} \theta\right)^{1 / 2}\right]
\end{aligned}
$$

with $\theta$ measured from the normal to the surface, the subscript $E$ denoting the electric field vector in the plane of incidence, subscript $\mathrm{H}$ denoting the electric field vector perpendicular to the plane of incidence, and the subscript 1 denoting the subsurface material (concrete, steel, soil, etc.). Figures 22 to 26 are contour plots of the reflection coefficient magnitude in $\varepsilon_{\mathrm{r}}-\sigma$ space for select frequencies in the range of interest. The values of the contours (magnitude of $\mathrm{R}$ ) are not as important and are not shown. Rather, the curves are meant to convey that the reflection coefficients (both $R_{E}$ and $R_{H}$ ) for the range of incidence angles are mainly functions of $\varepsilon_{R}$ and are therefore displacement current dominated. The implication is that for frequencies greater than about $32 \mathrm{MHz}$ (or thereabouts), the precise definition of $\sigma$ is not as important as an accurate definition of $\varepsilon_{R}$.

The expressions used in the LSQ fits (Equations (13) and (14)) were plotted parametrically over the range of $0 \mathrm{MHz}$ to $1 \mathrm{GHz}$. A version of Figure 21 with the LSQ-fit curve is shown in Figure 27. The fit is reasonable for the purposes of understanding the impact of $\left\{\varepsilon_{r}, \sigma\right\}$. Clearly, the constitutive parameter values lead to strong displacement current 
$16 \mathrm{MHz}$

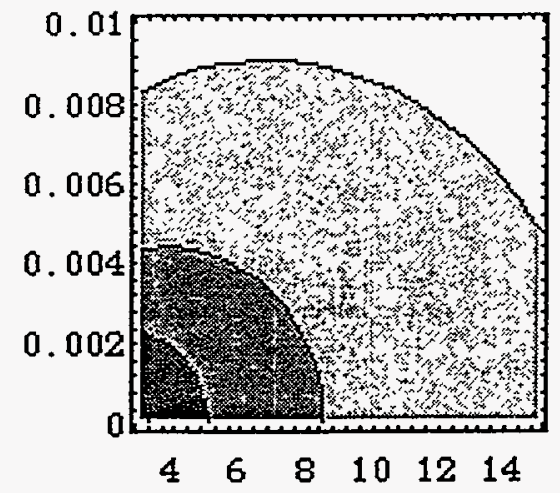

$64 \mathrm{MHz}$

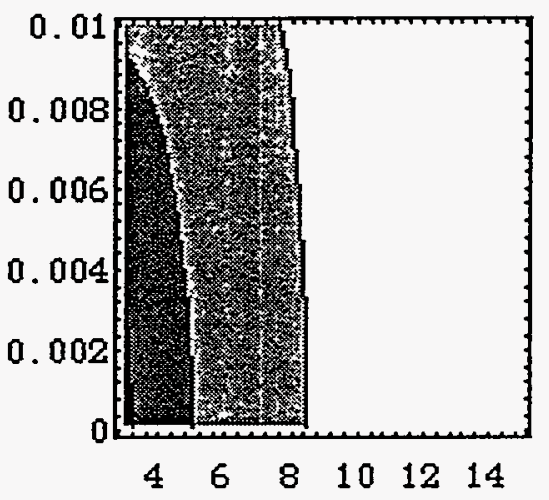

$256 \mathrm{MHz}$

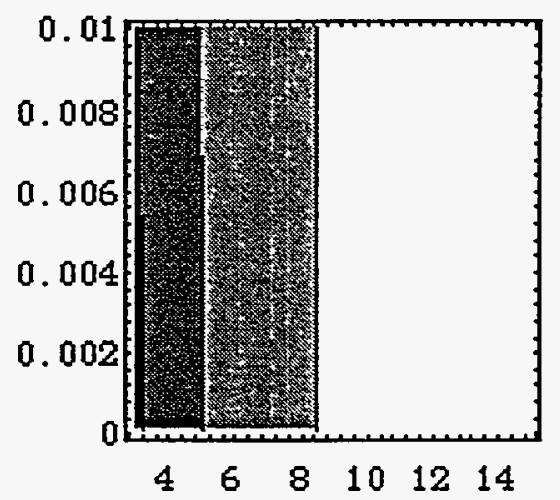

$32 \mathrm{MHz}$

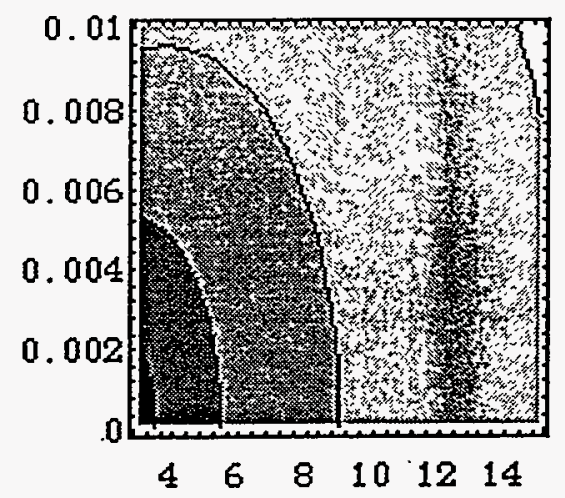

$128 \mathrm{MHz}$

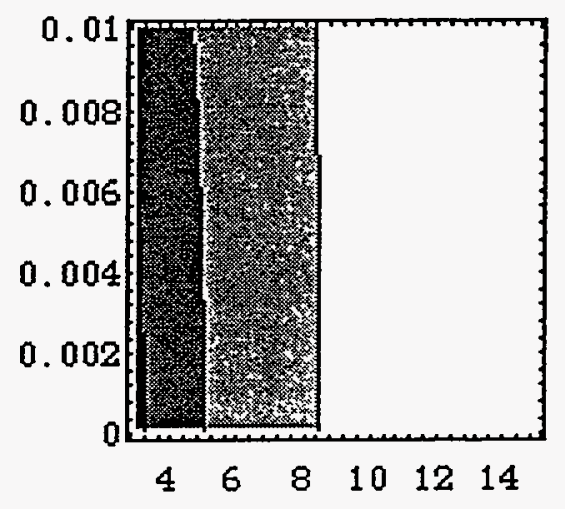

$512 \mathrm{MHz}$

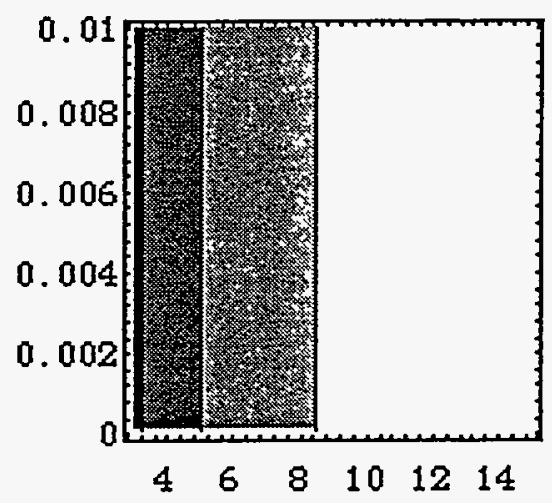

Figure 22. $R_{E}=R_{H}$ for normal incidence $(\theta=0)$ (Vertical axes are conductivity in Siemens $/ m$ and horizontal axes are relative dielectric constant) 

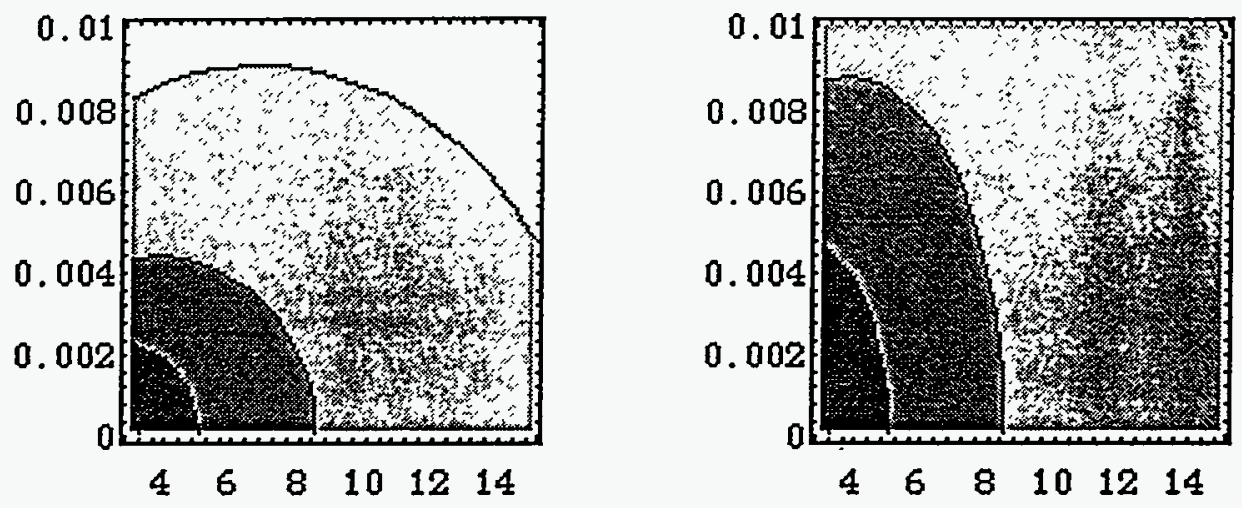

$64 \mathrm{MHz}$

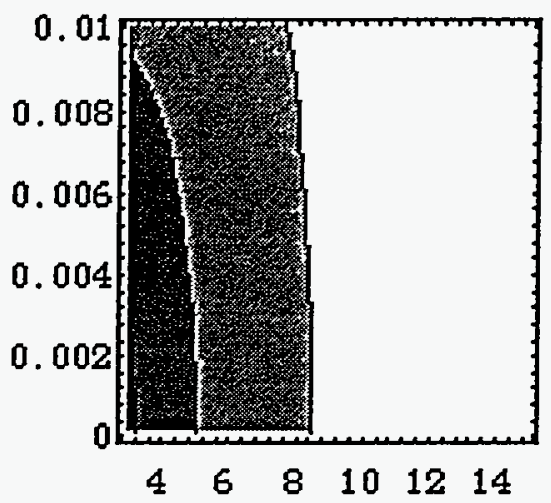

$128 \mathrm{MHz}$

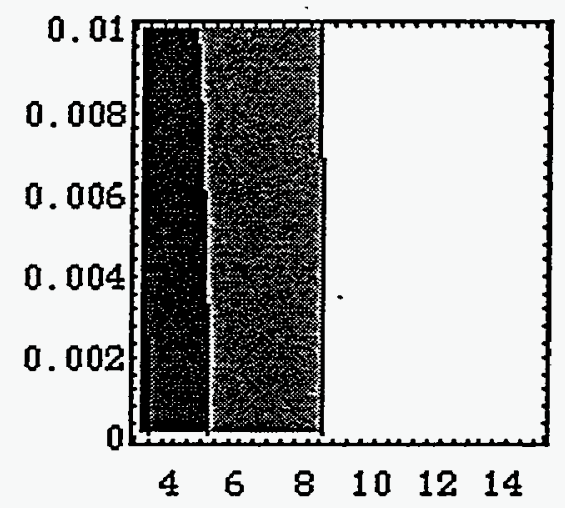

$256 \mathrm{MHz}$

$512 \mathrm{MHz}$
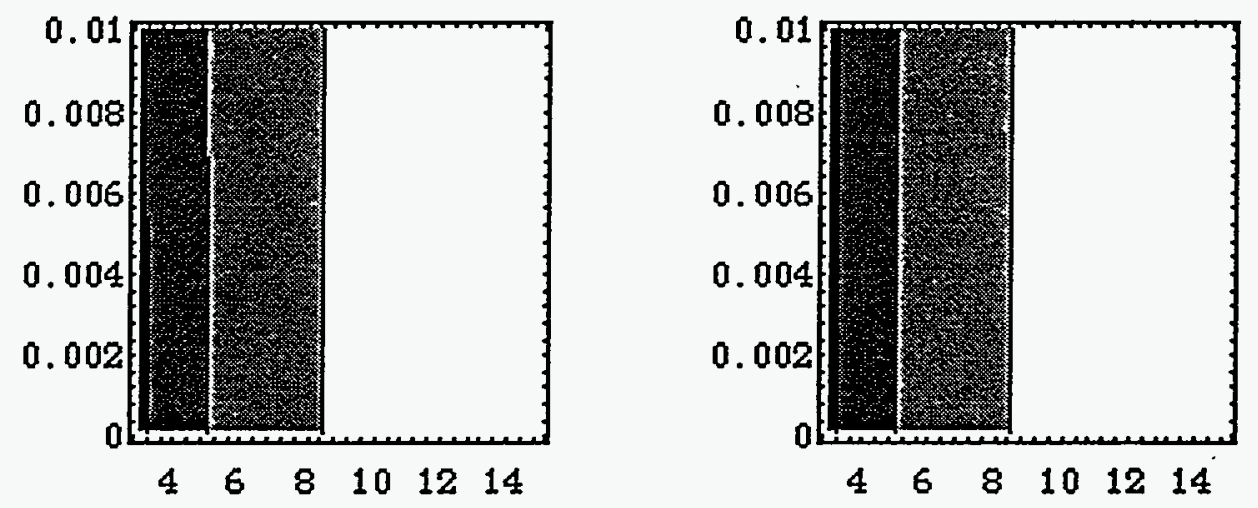

Figure 23. $R_{H}$ for incidence at 45 degrees (Vertical axes are conductivity in Siemens/m and horizontal axes are relative dielectric constant) 
$16 \mathrm{MHz}$

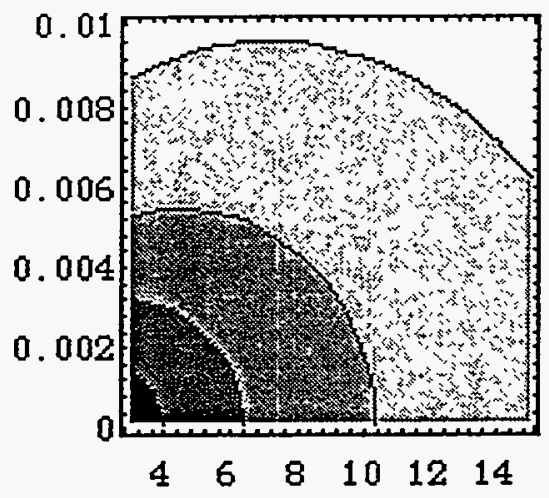

$64 \mathrm{MHz}$

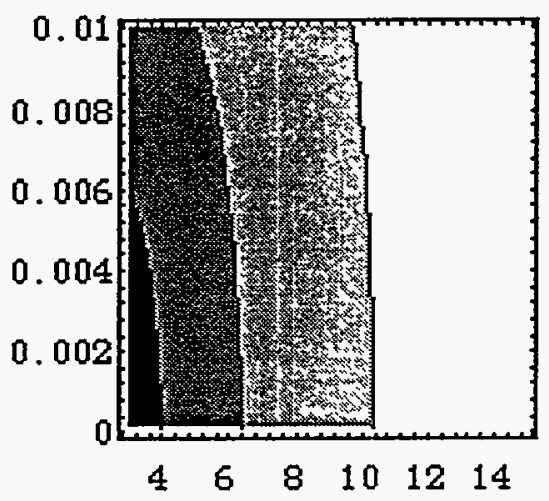

$256 \mathrm{MHz}$

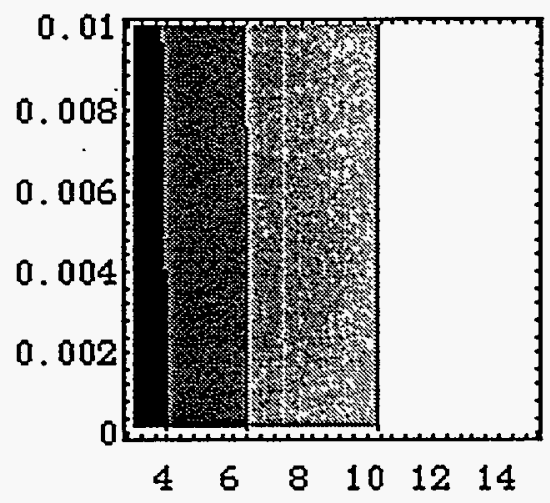

$32 \mathrm{MHz}$

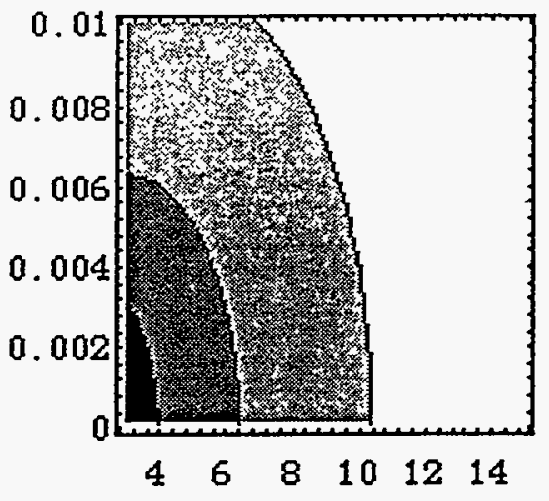

$128 \mathrm{MHz}$

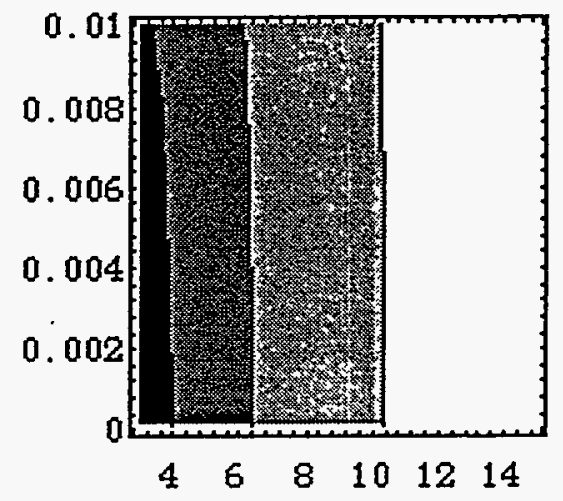

$512 \mathrm{MHz}$

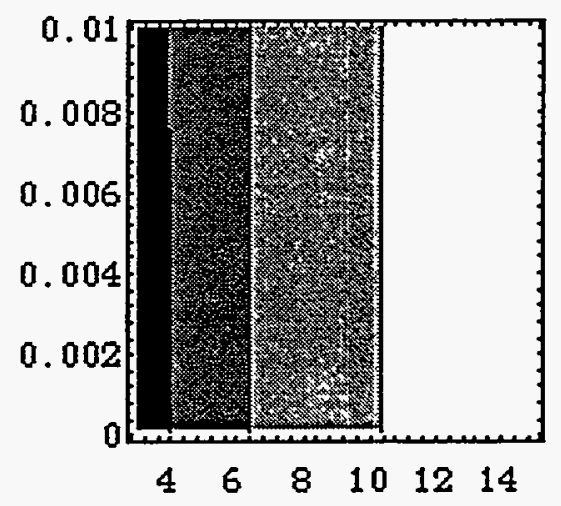

Figure 24. $R_{E}$ for incidence at 45 degrees (Vertical axes are conductivity in Siemens/m and horizontal axes are relative dielectric constant) 
$16 \mathrm{MHz}$

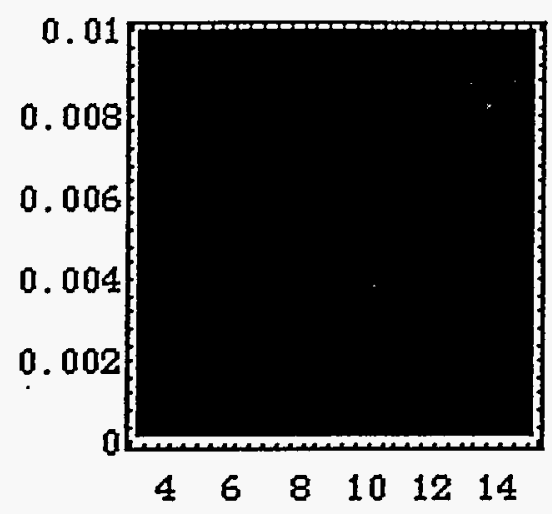

$64 \mathrm{MHz}$

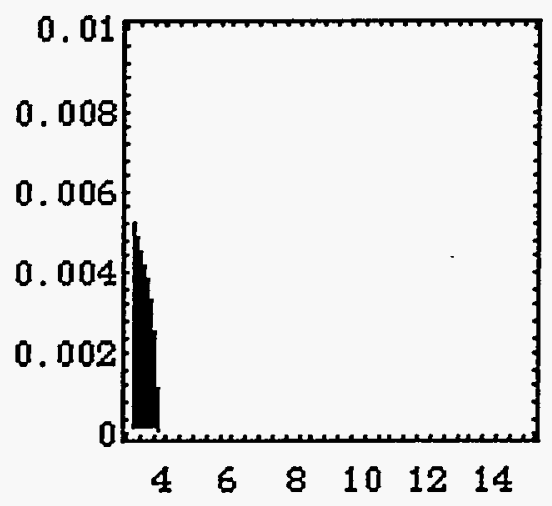

$256 \mathrm{MHz}$

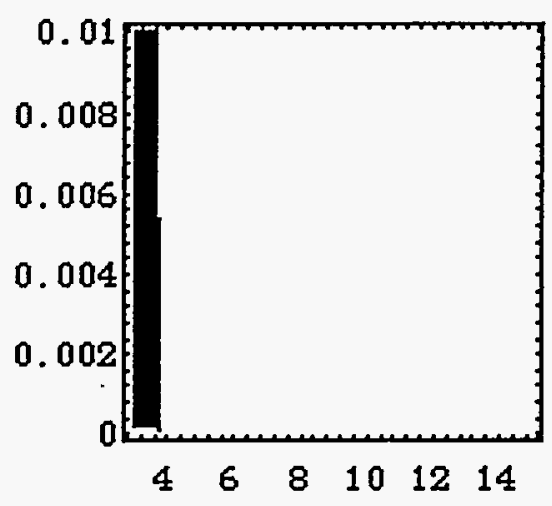

$32 \mathrm{MHz}$

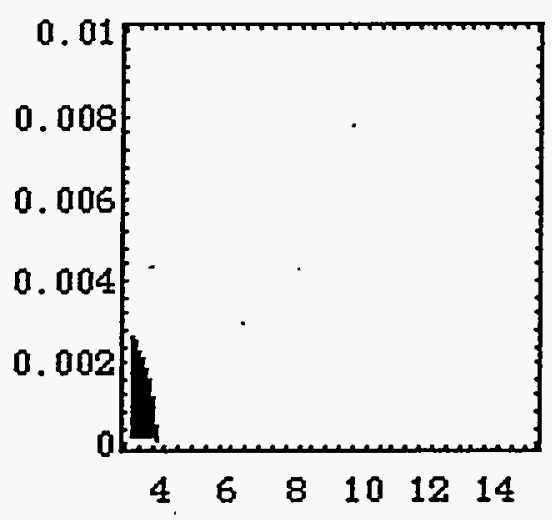

$128 \mathrm{MHz}$

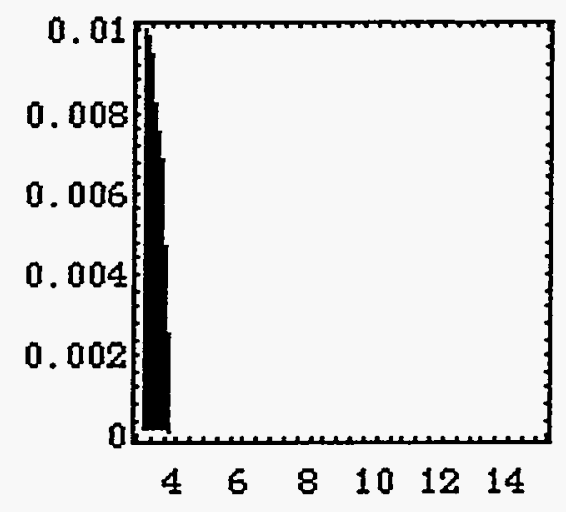

$512 \mathrm{MHz}$

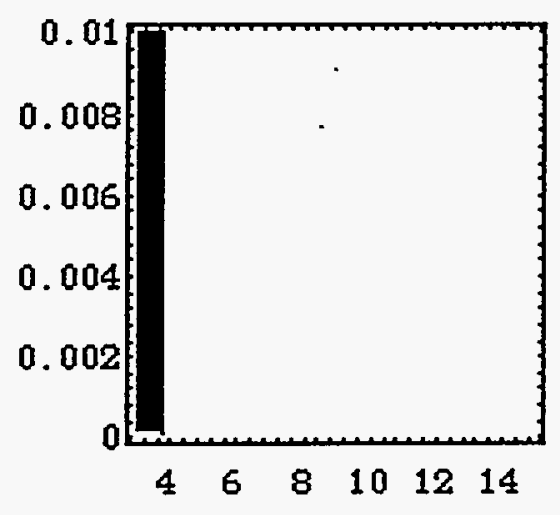

Figure 25. $R_{H}$ for grazing angle of incidence (85 degrees from normal incidence) (Vertical axes are conductivity in Siemens/m and horizontal axes are relative dielectric constant) 
$16 \mathrm{MHz}$

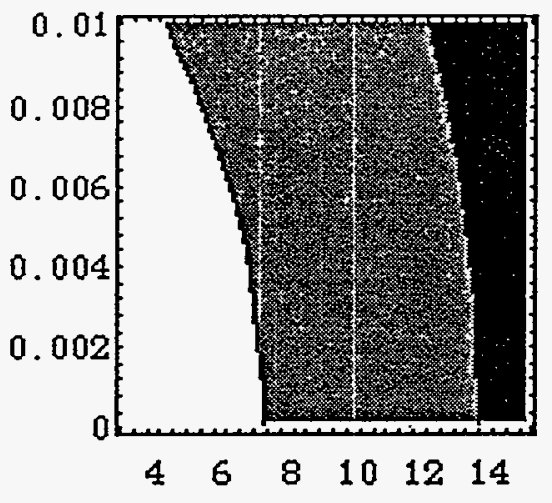

$64 \mathrm{MHz}$

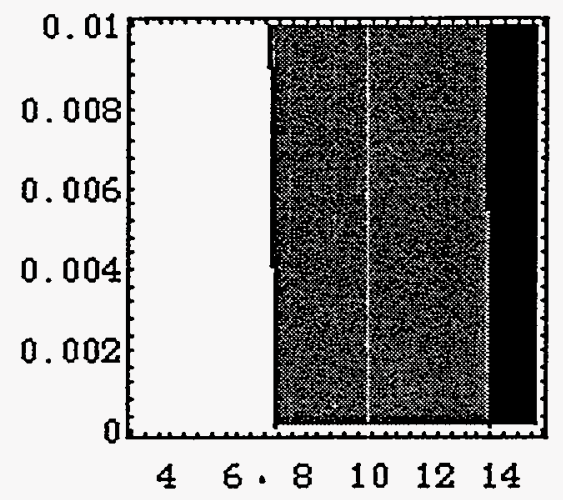

$256 \mathrm{MHz}$

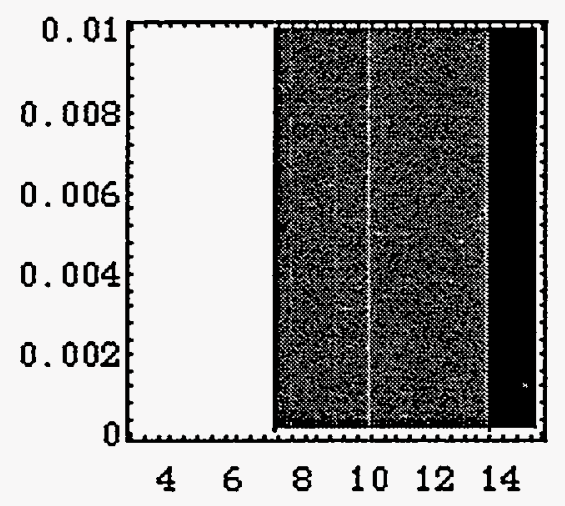

$32 \mathrm{MHz}$

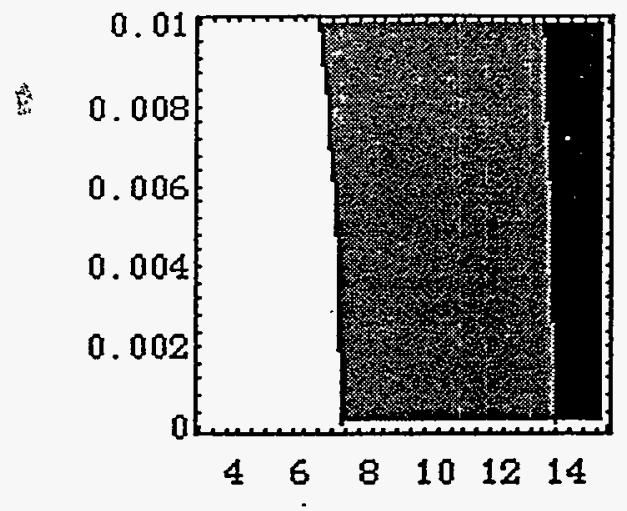

$128 \mathrm{MHz}$

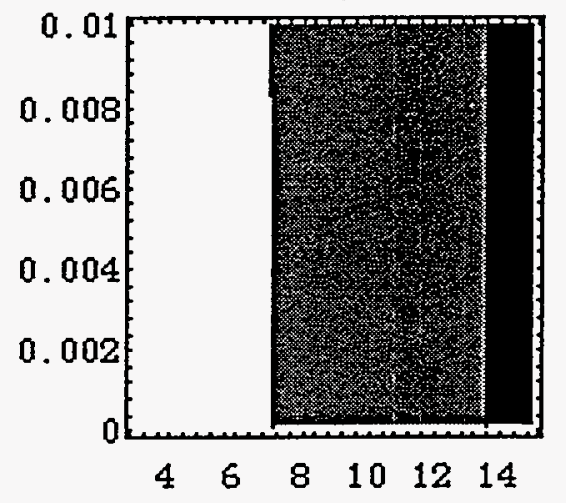

$512 \mathrm{MHz}$

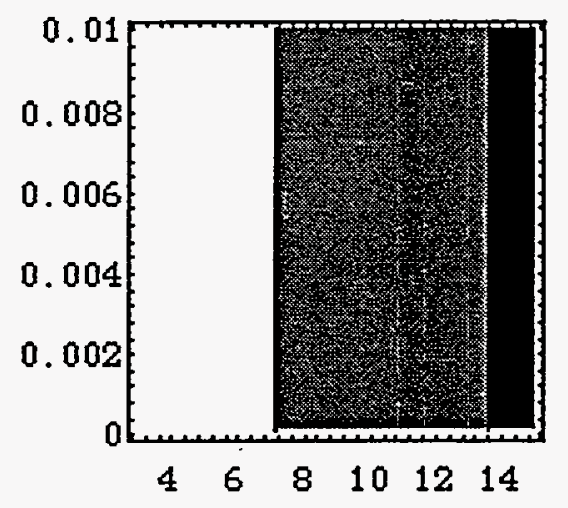

Figure 26. $R_{E}$ for grazing angle of incidence ( 85 degrees from normal incidence) (Vertical axes are conductivity in Siemens/m and horizontal axes are relative dielectric constant) 


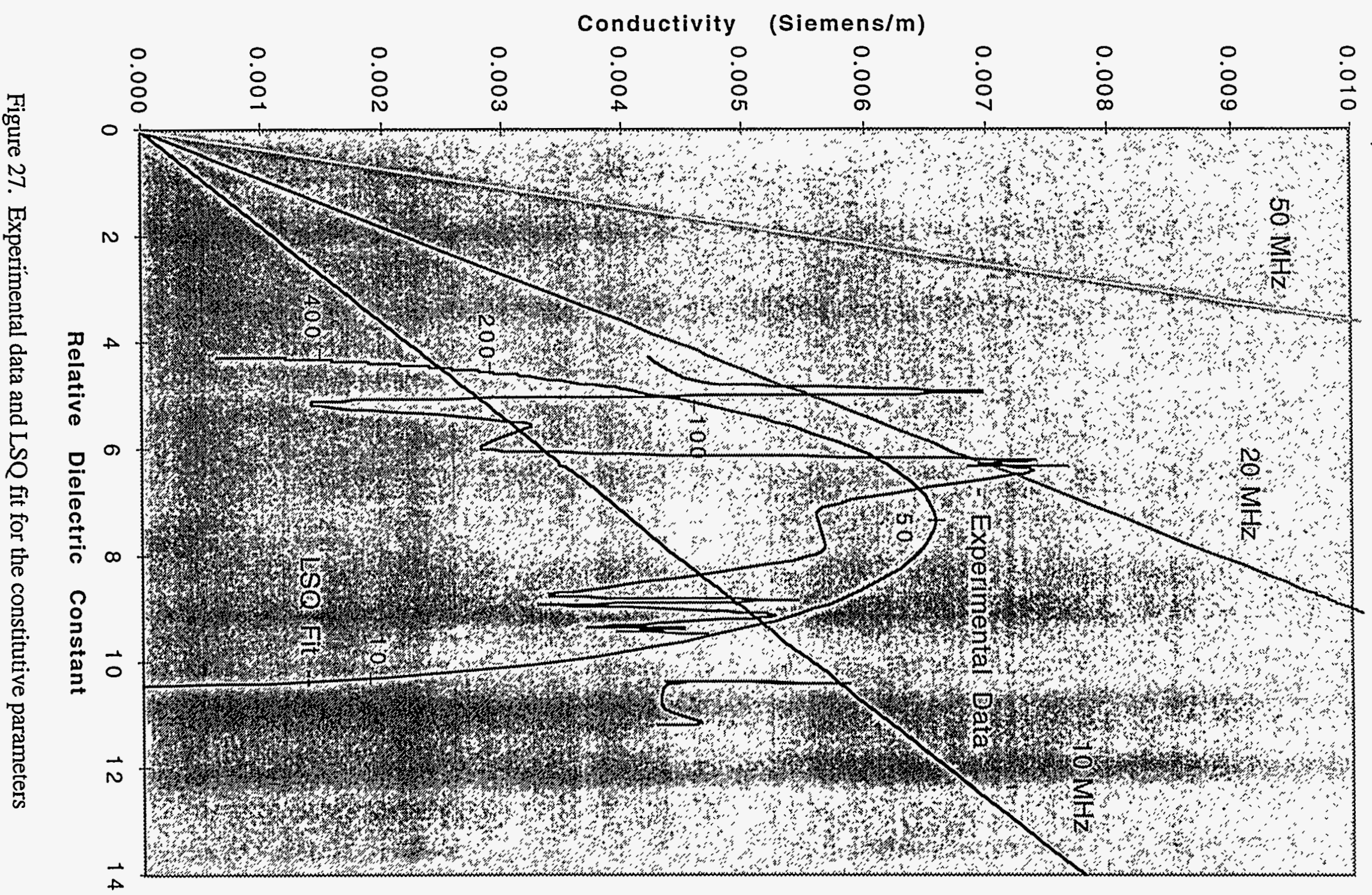


effects and the system is generally displacement current dominated. Plots of the relative dielectric constant and conductivity are shown in Figures 28 and 29 for frequencies up to about $300 \mathrm{MHz}$ to illustrate that the region of variability of the parameters is rather confined.

Another indicator of the importance of conduction or displacement current effects is the index of refraction for the medium, i.e.,

$$
\mathrm{n}=\left[\varepsilon_{\mathrm{r}}+\mathrm{i} \sigma /\left(\omega \mathrm{e}_{0}\right)\right]^{1 / 2}
$$

Clearly, $\sigma$ becomes unimportant when $\mathrm{n}$ can be addequately represented by

$$
\mathrm{n} \sim\left[\varepsilon_{\mathrm{r}}\right]^{1 / 2}
$$

Figure 30 shows curves representing the magnitude of Equations (19) and (20) and illustrates that above about $40 \mathrm{MHz}$, the differences are less than 3\%. Above about 150 $\mathrm{MHz}$, the index of refraction has effectively lost its dependence on frequency, and above $100 \mathrm{MHz}$, it has lost its strong dependency on $\sigma$. The index of refraction is, however, strongly affected by the values used for the relative dielectric constant throughout the entire frequency range.

Finally, the magnitude of the reflection coefficient was considered as a measure of the importance of $\sigma$. For our purposes, only the normal incidence coefficient was evaluated using the index of refraction in Equation (19) and the approximation $(\sigma=0)$ given by Equation (20). Figure 31 is a plot of the two representations for $R_{E}=R_{H}$ for normal incidence. The deviation in the magnitude is less than about $5 \%$ for all frequencies. The reflection coefficient is effectively independent of frequency above $150 \mathrm{MHz}$ and nearly so above $80 \mathrm{MHz}$. It is nearly independent of $\sigma$ above $50 \mathrm{MHz}$.

The previous discussions have considered only the relative importance of $\varepsilon_{\mathrm{r}}$ and $\sigma$ in the determination of parameters defining wave propagation in the medium. In the following we consider the magnitude of the index of refraction evaluated from the constitutive parameters resulting from our measurements, and look at its comparison to the magnitude of the index obtained from our models for the constitutive parameters based on LSQ fits to our data. Figure 32 is a plot of the magnitude of $n$ versus frequency using the model for $\varepsilon_{\mathrm{r}}$ and $\sigma$ as compared to the magnitude as determined from $\varepsilon_{\mathrm{r}}$ and $\sigma$ obtained from processing 


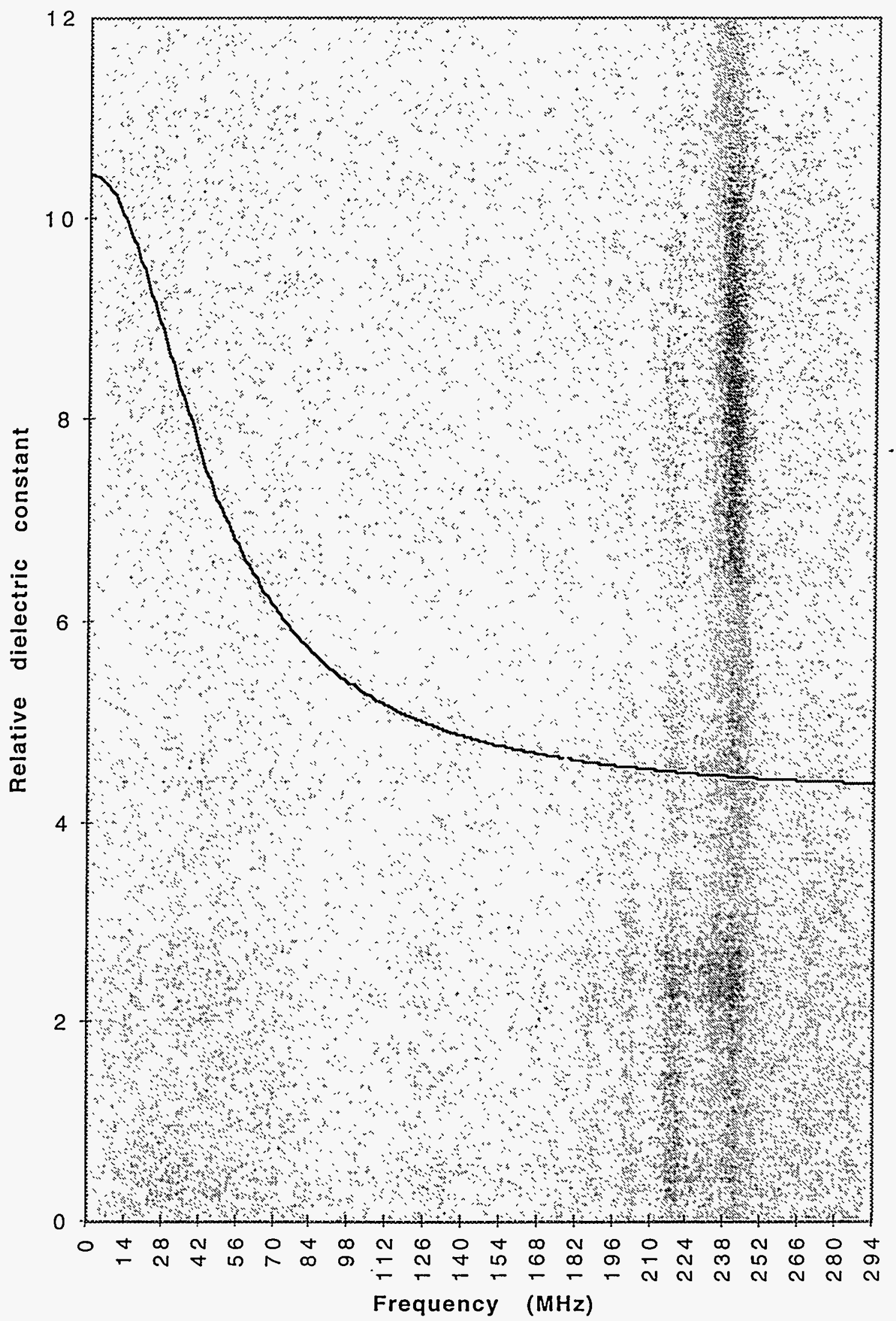

Figure 28. Relative dielectric constant versus frequency per Equation (13) 


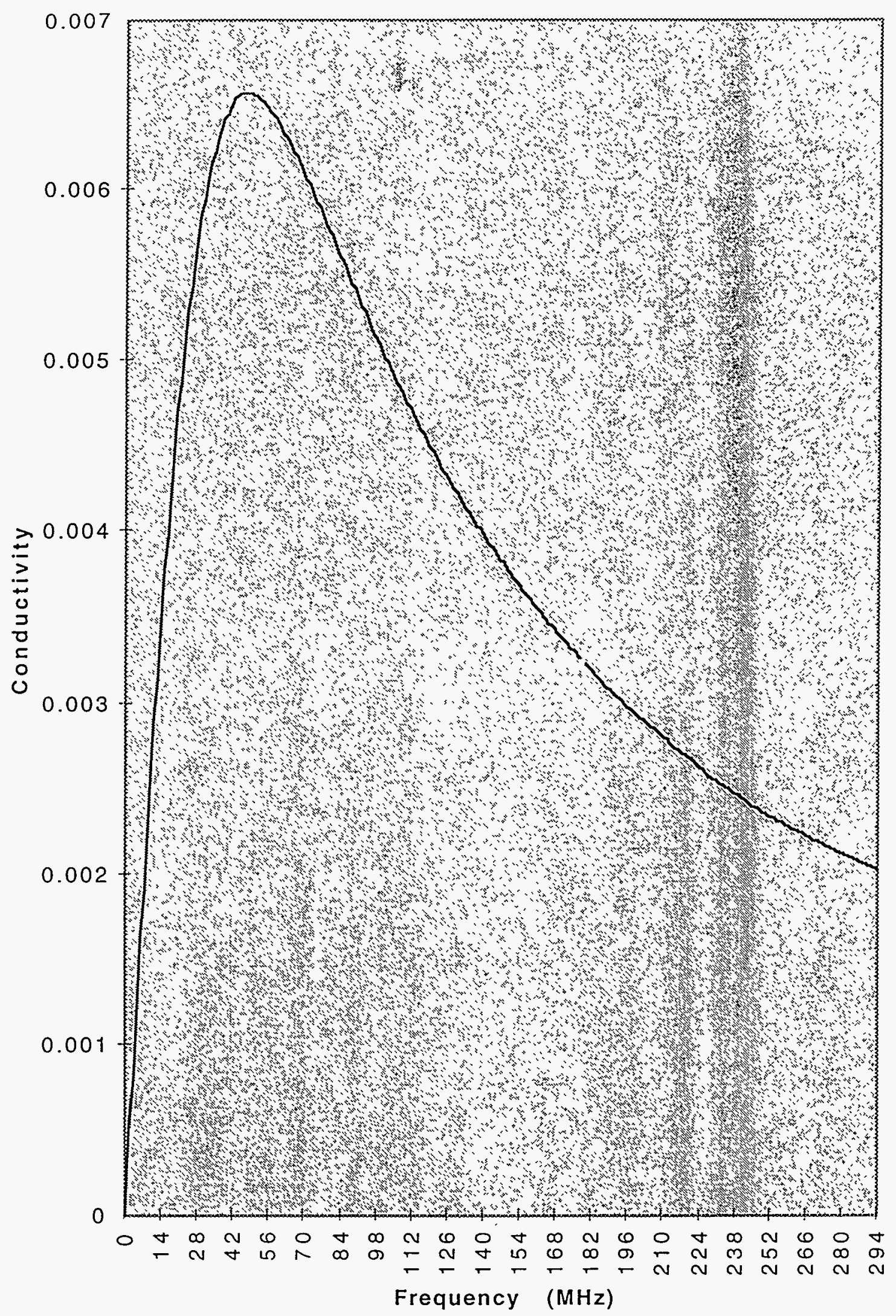

Figure 29. Conductivity (Siemens/m) versus frequency per Equation (14) 


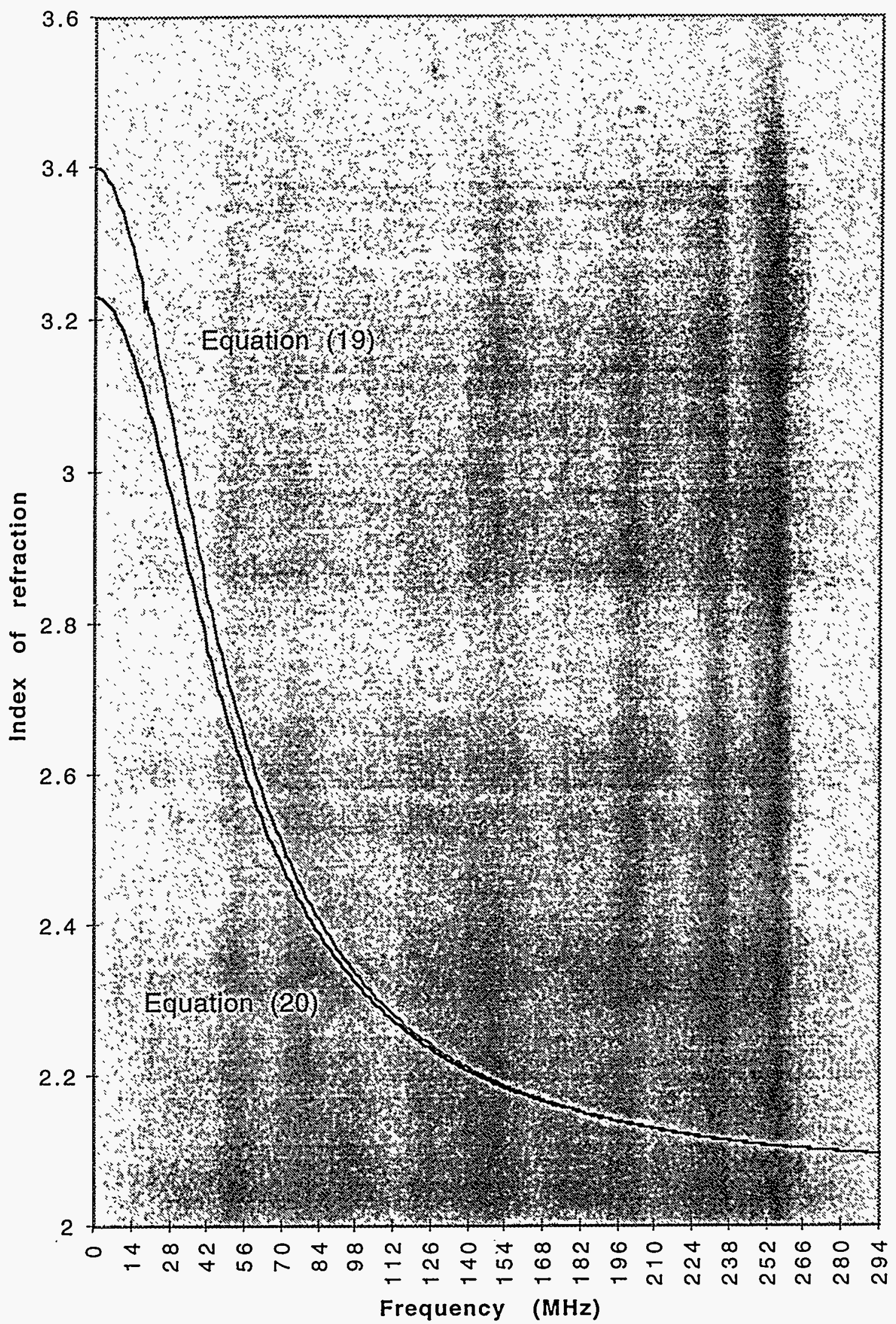

Figure 30. Two representations for the index of refraction versus frequency 


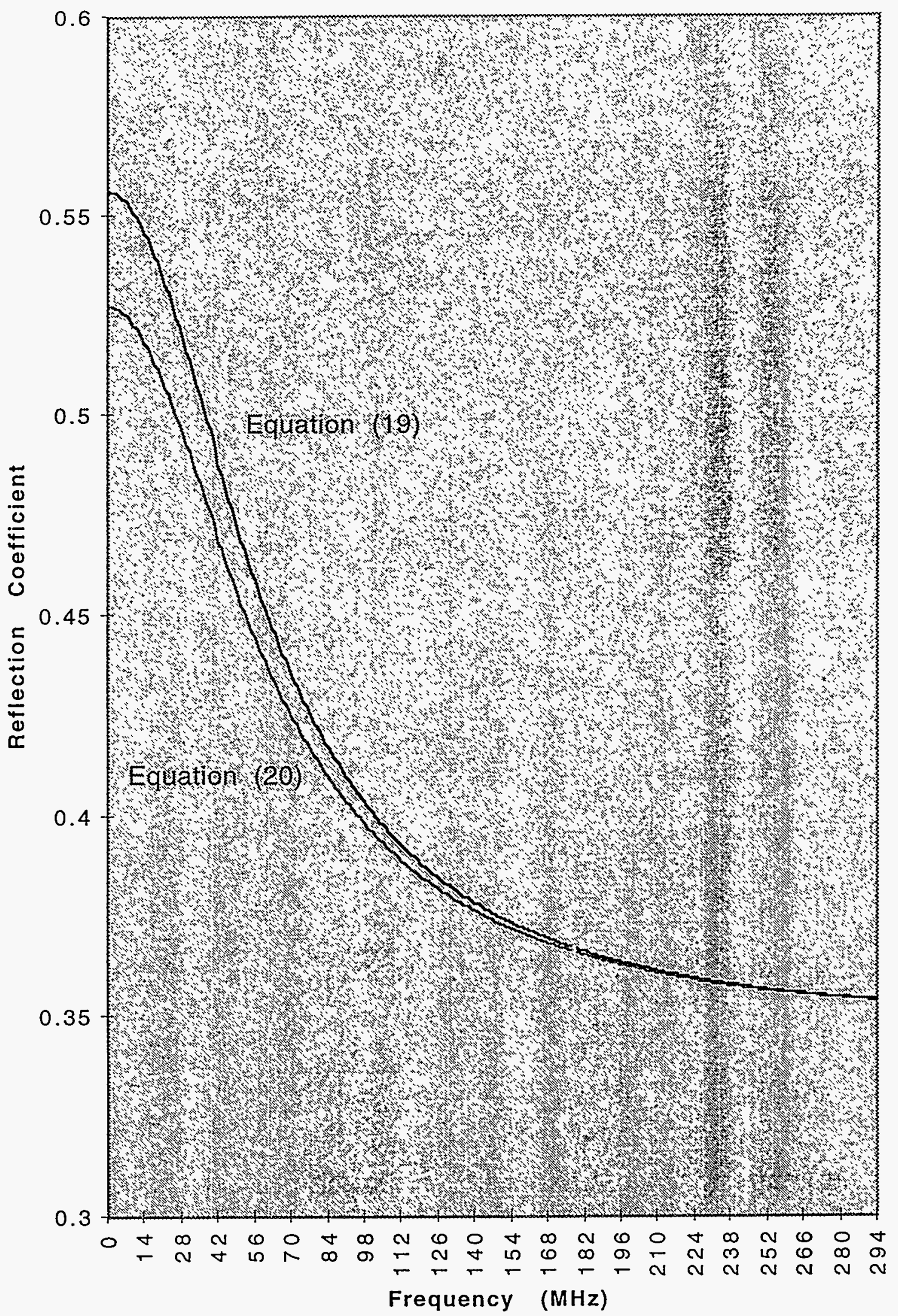

Figure 31. Two representations for the normal incidence reflection coefficient vs. frequency 


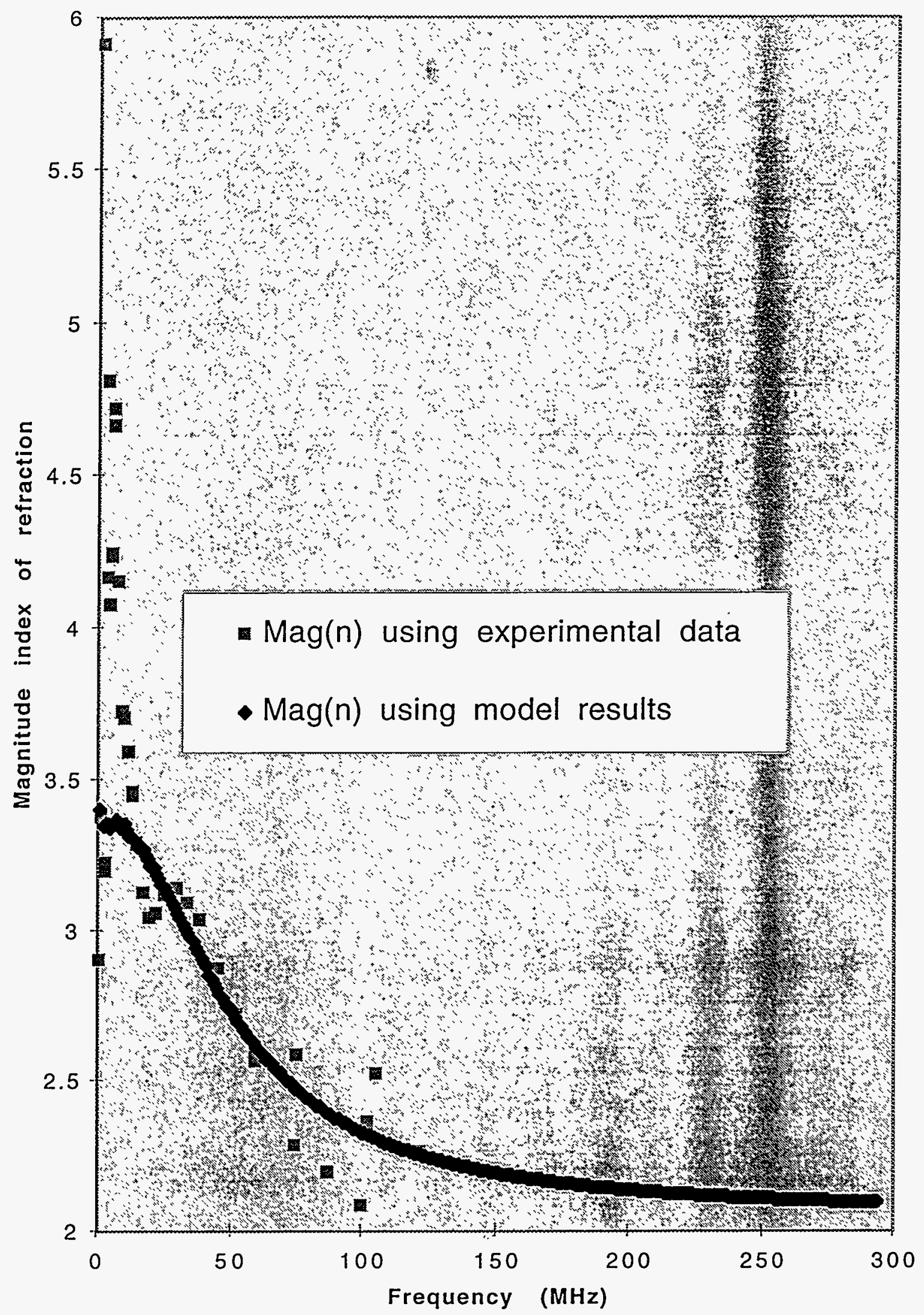

Figure 32. Magnitude of index of refraction as obtained from raw data and from models 
our experimental data. The agreement over the range from $18 \mathrm{MHz}$ or so through 126 $\mathrm{MHz}$ is quite reasonable and provides some confidence in using the models for prediction at higher frequencies. The apparent discrepancy between the model and the experimentallybased results may be in part due to the difficulty in determining $\alpha$ and $\beta$, and ultimately $\varepsilon_{\mathrm{r}}$ and $\sigma$, from the experimental data.

The relative sensitivity of the reflection coefficient to variations in the index of refraction for the special condition where $\mu_{1}=\mu_{0}$ will be considered to provide some indication of the effects of errors in the process. A full error analysis is beyond the scope of this report. With

$$
\begin{aligned}
& R_{H}=\left[\cos \theta-\left(n^{2}-\sin ^{2} \theta\right)^{1 / 2}\right] /\left[\cos \theta+\left(n^{2}-\sin ^{2} \theta\right)^{1 / 2}\right] \\
& R_{E}=\left[n^{2} \cos \theta-\left(n^{2}-\sin ^{2} \theta\right)^{1 / 2}\right] /\left[n^{2} \cos \theta+\left(n^{2}-\sin ^{2} \theta\right)^{1 / 2}\right]
\end{aligned}
$$

consideration will be given to

$$
\begin{aligned}
& \left(1 / R_{H}\right)\left(\partial R_{H} / \partial n\right)=2 n \cos \theta\left(n^{2}-1\right)^{-1}\left(n^{2}-\sin ^{2} \theta\right)^{-1 / 2} \\
& \left(1 / R_{E}\right)\left(\partial R_{E} / \partial n\right)=2 n \cos \theta\left(n^{2}-\sin ^{2} \theta\right)^{-1 / 2}\left(n^{2}-2 \sin ^{2} \theta\right) /\left(n^{4} \cos ^{2} \theta-n^{2}+\sin ^{2} \theta\right)
\end{aligned}
$$

An approximation for the fractional change due to changes in $\mathrm{n}$ is

$$
\begin{aligned}
& \left|\Delta R_{H} / R_{H}\right|=2\left|n \cos \theta\left(n^{2}-1\right)^{-1}\left(n^{2}-\sin ^{2} \theta\right)^{-1 / 2} \Delta n\right| \\
& \left|\Delta R_{E} / R_{E}\right|=2 \mid n \cos \theta\left(n^{2}-\sin ^{2} \theta\right)^{-1 / 2}\left(n^{2}-2 \sin ^{2} \theta\right) /\left(n^{4} \cos ^{2} \theta-n^{2}+\sin ^{2} \theta\right) \Delta n
\end{aligned}
$$

Over the range from 20 to $120 \mathrm{MHz}$ (the range of the experimental data in Figure 32), we find that $\left|\Delta R_{H} / R_{H}\right| \leq 0.6|\Delta n|$ and $\left|\Delta R_{E} / R_{E}\right| \leq 1.5|\Delta n|$. The maximum value in the latter result is an overestimated bound except in the vicinity of the Brewster angle. Removed from the Brewster angle, the result for $R_{E}$ is in keeping with the result for $R_{H}$.

In view of the observations made in this section, the constitutive parameters determined from experimental data over a limited frequency range and the models based on these limited data appear to yield reliable values outside the range of the useable experimental data. 


\section{Conclusions and Summary :}

\subsection{General Comments}

The approach taken herein has proven to be a viable method to determine the constitutive parameters of a medium using noninvasive techniques. There have not been reports in the literature of attempts to perform such constitutive parameter extraction other than in the form of passing comments [10]. The method presented here is based on sound theoretical bases. Some approximations and assumptions have been made in the analysis, e.g., that $\mu_{1}=\mu_{2}=\mu_{0}, \sigma_{\text {wire }}=\infty$, homogeneity of the ground, absence of reflected waves on the wire (effective infinite length), perturbations of the measured current distributions by the measuring system are minimal, and the calibration factors for the measuring system are accurate. The method, while not necessarily robust, has yielded valuable information regarding the characteristics of the ground pad and has uncovered some of the capabilities and shortcomings of the approach. Some comments will be included in a later section.

Since the test program was conducted in a one-week period which did not allow for repeats of the data acquisition/analysis process or even for modifications of the process given observations based on analysis of the data, improvements in the experimental setup to permit improved estimates of the constitutive parameters have not been possible Nonetheless, a reasonable representation of the constitutive parameters of the reinforced concrete pad in the LESLI facility at the Phillips Lab has been achieved.

The constitutive parameters determined by this process are to be used in a program where the realistic ground is to be part of a computer model used in code validation. The test object, a Boeing 757 parked on a reinforced concrete pad, has a fuselage which is seven or more feet above the ground and the test points, except for a top-mounted external blade antenna, are located internal to the fuselage. It has always been expected that the ground will have its most pronounced effect on the structure of the field illuminating the airplane and will most likely have little effect on secondary scattering where the illuminating wave impinges on the airplane, scatters, impinges on the ground, scatters, and impinges on the airplane (thus "perturbing" the illuminating field). In a full wave analysis, this separation or consideration of the order of scattering is not an issue. We only make these comments to reflect on the relative importance of the ground characteristics in the ultimate solution. 


\subsection{Data Summary}

The experimental data in the form of current distributions on a wire over ground were collected over a frequency range from approximately $1 \mathrm{MHz}$ to $1 \mathrm{GHz}$ at 90 spatial points spaced at $30 \mathrm{~cm}$ intervals. Reasonably accurate estimates of attenuation and phase constants were obtained for frequencies between $10 \mathrm{MHz}$ and $126 \mathrm{MHz}$. Below this range, the spatial extent of sampling did not permit accurate estimates of the attenuation constant while beyond this frequency range, the sampling density and the limited support of the transmission line mode did not permit accurate estimates of the phase and attenuation constants, respectively. Over the frequency range, the constants led to estimates of $\varepsilon_{\mathrm{r}}$ and $\sigma$ which were "reasonable" and within the range of physical realizability. Outside the range, the estimates for the constitutive parameters were decidedly less reliable. The estimates from the reliable range were used in a least squares fit to the Debye equations to yield expressions for the constitutive parameters and their average values over range $\left\{f_{1}, f_{2}\right)$ :

$$
\begin{aligned}
& \varepsilon_{\mathrm{r}}=4.2418+6.1943 /\left(1+1.129 \cdot 10^{-17} \cdot \omega^{2}\right) \\
& \sigma=4.416 \cdot 10^{-11} \cdot \omega /\left(1+1.354 \cdot 10^{-17} \cdot \omega^{2}\right) \\
& <\varepsilon_{\mathrm{r}}\left(\mathrm{f}_{1}, \mathrm{f}_{2}\right)>=4.24+2.93 \cdot 10^{8} \cdot\left[\tan ^{-1}\left(2.11 \cdot 10^{8} \cdot \mathrm{f}_{2}\right)-\tan ^{-1}\left(2.11 \cdot 10^{8} \cdot \mathrm{f}_{1}\right)\right] /\left(\mathrm{f}_{2}-\mathrm{f}_{1}\right) \\
& <\sigma\left(\mathrm{f}_{1}, \mathrm{f}_{2}\right)>=2.595 \cdot 10^{5} \cdot \operatorname{Ln}\left[\left(1+5.29 \cdot 10^{-16} \cdot \mathrm{f}_{2}{ }^{2}\right) /\left(1+5.29 \cdot 10^{-16} \cdot \mathrm{f}_{1}{ }^{2}\right)\right] /\left(\mathrm{f}_{2}-\mathrm{f}_{1}\right)
\end{aligned}
$$

Analysis of the experimental data allowed the identification of areas where the displacement current effects dominated. Certainly, displacement current effects were dominant above 50 $\mathrm{MHz}$ and it was evident from the reflection coefficient analysis that it was effectively independent of conductivity above $50 \mathrm{MHz}$ and effectively independent of frequency above $100 \mathrm{MHz}$. Thus concern regarding the conductivity was secondary as its impact would be felt only below $50 \mathrm{MHz}$.

\subsection{Concerns and Suggested Future Work on the Process}

As mentioned previously, time and operational constraints did not permit a more in-depth study of the constitutive parameters and the technique used for their determination. In the future, this technique deserves an extended investigation as it represents a viable method for noninvasive determination of $\varepsilon_{\mathrm{r}}$ and $\sigma$. Specifically, several issues should be carefully studied to assess their impact on accuracy. In the following, several suggestions are made for future uses of the method and several areas are recommended for study. 


\section{Spatial Sampling}

Prior to testing, an analysis was executed to bound the sampling density required to get reliable data over the interval $10 \mathrm{MHz}$ to $400 \mathrm{MHz}$ subject to the operational constraints of time and equipment availability. At the low frequencies it was anticipated that the difficulties would arise from the total attenuation that would be experienced over the entire sampling range. At the high frequencies it was expected that the difficulty would arise from the length of the interval over which the transmission line mode was dominant and the number of samples in this interval. In both cases the accuracy of the measurement system was a concern as was its dynamic range. While relative measurements $\left(\mathrm{I}(\mathrm{x}) /\left(\mathrm{x}_{0}\right)\right)$ were adequate for our purposes, the system was limited by its inability to make accurate measurements of either component below $-80 \mathrm{dBm}$ or thereabouts. It was originally felt that sampling at four samples per wavelength was adequate so that the method could produce high quality results at $250 \mathrm{MHz}$ at a sample spacing of $30 \mathrm{~cm}$. Also, the total sampling range was 26.7 meters and it was estimated that sampling over a wavelength at low frequencies (approximately $11 \mathrm{MHz}$ ) would be adequate to assess the attenuation constant. In the presence of noise, reflections, and other fluctuations in the current distribution, the determination of attenuation constant at low frequencies was difficult. The attenuation observed over the entire sampling range was small. At high frequencies, these problems were compounded by the small interval over which the transmission line mode was dominant, the dynamic range of the measurement system, and the small number of samples per wavelength .

In future exercises, it would be beneficial to start the sampling closer to the source to increase the reference signal level and to maximize the length over which the transmission line mode dominates. This will help to extend the high frequency limit. Also, the sampling density should be increased in the high frequency regime in order to generate more samples.per wavelength. For the low frequency range, the length over which sampling takes place should be made as large as possible so that the current is sampled over several wavelengths. In the face of limited resources, rather than having uniform sampling over the entire measurement range, it will be beneficial to sample more densely near the source for high frequency coverage and less densely for low frequency coverage. This suggests a nonuniform sampling scheme and may imply a logarithmic sampling rate with more density near the source and less farther away. 


\section{$\underline{\text { Reflections }}$}

The presence of reflections on the line must be minimized. The current distribution must be entirely that of a transmission line mode on an infinite line. The extraction of reflected waves from unknown locations could make the process difficult if not impossible. Proper loading of finite length lines or sufficient length could make this issue insignificant.

\section{$\underline{\text { Height }}$}

At present the impact of uncertainties on the accuracy of parameter estimates is unknown, in detail. Certainly the uncertainties here (plus or minus $1 / 8$ " at a height of $5 / 8$ ") have not caused major difficulties but the overall impact should be studied.

\section{System Effects}

The effects of the measurement probes, e.g., the current probes and their cables, on the accuracy of the current distribution measurement should be looked at in detail.

\section{Mathematical Issues}

The process, which is a classical inversion problem, may not be robust. Small errors in $\alpha$ and $\beta$ could lead to significant errors in $\varepsilon_{\mathrm{r}}$ and $\sigma$ although such problems were not overwhelming in this case. This aspect should be studied to lend a measure of usefulness to this technique. In the range of displacement current domination, when a consistent solution may not be available because of great uncertainties in $\sigma$ due to its limited impact on the current distribution, are there methods to obtain reasonable estimates of $\varepsilon_{\mathrm{r}}$ which would suffice? Are alternate methods available? Likewise, similar questions must be answered for the case when conduction current dominates. 


\section{References}

1) A. J. Poggio, R. A . Zacharias, S. T. Pennock, C. Avalle, R. Sharpe, K. Kunz, and C. Meissner, "NASA FBL/PBW Program NASA Boeing 757 HIRF Test Plan, Low Power On-the-Ground Tests," October, 1994

2) D. C. Chang and R. G. Olsen, "Excitation of an Infinite Antenna Above a Dissipative Earth," Radio Science, Vol. 10, No. 8,9, pp. 823-831, August-September 1975.

3) E. F. Kuester, D. C..Chang and R. G. Olsen, "Modal Theory of Long Horizontal Wire Structures Above the Earth, 2, Properties of Discrete Modes," Radio Science, Vol. 13, No. 4, pp. 615-623, July-August 1978.

4) R. G. Olsen, E. F. Kuester and D. C. Chang, "Modal Theory of Long Horizontal Wire Structures Above the Earth, 1, Excitation," Radio Science, Vol. 13, No. 4, pp. 605613, July-August 1978.

5) G. J. Burke, "A Comparison of Solutions for Wires Over Ground", 8th Annual Review of Progress in Applied Computational Electromagnetics, 16-20 March, 1992.

6) G. J. Burke and A. J. Poggio, "Numerical Elecromagnetics Code (NEC) - Method of Moments" Lawrence Livermore National Laboratory, Rept. UCID - 18834, January 1981.

7) R. W. P. King and G. S. Smith, Antennas in Matter, MTT Press, 1981

8) R. S. Elliott, Electromagnetics, McGraw-Hill, 1966

9) C. Kittel, Quantum Theory of Solids, John Wiley, 1963

10) B. Rama Rao and D. N. Jones, "Characterization of a High Frequency Beverage Antenna Using a Fiber-Optic Measurement Technique", Antennas and Propagation Symposium, June 24 - 28, 1991, London; Ontario 